\title{
On the existence of blowing-up solutions for a mean field equation ${ }^{\text {th }}$
}

\author{
Pierpaolo Esposito $^{\mathrm{a}, *}$, Massimo Grossi $^{\mathrm{b}}$, Angela Pistoia $^{\mathrm{c}}$ \\ a Dipartimento di Matematica, Università degli Studi "Roma Tre”, Largo S. Leonardo Murialdo, 1, 00146 Roma, Italy \\ b Dipartimento di Matematica, Università di Roma "La Sapienza", P.le A. Moro, 00166, Roma, Italy \\ c Dipartimento di Metodi e Modelli Matematici, Università di Roma "La Sapienza", Via Scarpa, 16, 00166, Roma, Italy
}

Received 23 February 2004; received in revised form 6 June 2004

\section{Abstract}

In this paper we construct single and multiple blowing-up solutions to the mean field equation:

$$
\begin{cases}-\Delta u=\lambda \frac{V(x) \mathrm{e}^{u}}{\int_{\Omega} V(x) \mathrm{e}^{u}} & \text { in } \Omega, \\ u=0 & \text { on } \partial \Omega,\end{cases}
$$

where $\Omega$ is a smooth bounded domain in $\mathbb{R}^{2}, V$ is a smooth function positive somewhere in $\Omega$ and $\lambda$ is a positive parameter. (C) 2005 L'Association Publications de l'Institut Henri Poincaré. Published by Elsevier B.V. All rights reserved

\section{Résumé}

Dans ce papier nous construisons des solutions qui explosent pour l'équation de champ moyen :

$$
\begin{cases}-\Delta u=\lambda \frac{V(x) \mathrm{e}^{u}}{\int_{\Omega} V(x) e^{u}} & \text { in } \Omega \\ u=0 & \text { on } \partial \Omega\end{cases}
$$

où $\Omega$ est un domaine borné dans $\mathbb{R}^{2}, V$ est une fonction positive dans $\Omega$ et $\lambda$ est un paramètre positif. (C) 2005 L'Association Publications de l'Institut Henri Poincaré. Published by Elsevier B.V. All rights reserved

\footnotetext{
is The first and second authors are supported by M.U.R.S.T., project "Variational methods and nonlinear differential equations". The third author is supported by M.U.R.S.T., project "Metodi variazionali e topologici nello studio di fenomeni non lineari”.

* Corresponding author.

E-mail addresses: esposito@mat.uniroma3.it (P. Esposito), grossi@mat.uniroma1.it (M. Grossi), pistoia@dmmm.uniroma1.it (A. Pistoia).
} 


\section{Introduction}

We consider the problem:

$$
\begin{cases}-\Delta u=\lambda \frac{V(x) \mathrm{e}^{u}}{\int_{\Omega} V(x) \mathrm{e}^{u}} & \text { in } \Omega \\ u=0 & \text { on } \partial \Omega\end{cases}
$$

where $\Omega$ is a smooth bounded domain in $\mathbb{R}^{2}, V$ is a smooth function positive somewhere in $\Omega$ and $\lambda \in \mathbb{R}$ is a positive parameter.

This equation occurs in various context such as: conformal geometry (cf. [2]), statistical mechanics (cf. [9,10]) and several other area of applied mathematics (cf. e.g. [6,13,23,34]). In statistical mechanics it is referred as the "mean field equation". In all those contexts, there is a definite interest to construct solutions which "blow-up" and "concentrate" at a set of given points, whose location carries relevant information about the geometrical/physical properties of the problem under exam.

We are interested in finding solutions $u_{\lambda}$ to (1.1) which blow-up at $k$ different points $q_{1}, \ldots, q_{k}$ in $\Omega^{\prime}$ along a sequence $\lambda \rightarrow 8 \pi k$, in the following sense:

$$
\lambda \frac{V(x) \mathrm{e}^{u_{\lambda}}}{\int_{\Omega} V(x) \mathrm{e}^{u_{\lambda}}} \rightarrow 8 \pi \sum_{i=1}^{k} \delta_{q_{i}} \text { in the sense of measures in } \bar{\Omega},
$$

where $\delta_{p}$ denotes the Dirac measure at $p$. Here $\Omega^{\prime}=\{q \in \Omega: V(q)>0\}$.

In order to state some new and old results it is useful to introduce some notation. Let $G_{\Omega}$ denote the Green's function of $-\Delta$ with Dirichlet boundary condition on $\Omega$, namely for any $y \in \Omega$ it holds

$$
\begin{cases}-\Delta_{x} G_{\Omega}(x, y)=\delta_{y}(x) & \text { if } x \in \Omega, \\ G_{\Omega}(x, y)=0 & \text { if } x \in \partial \Omega\end{cases}
$$

and let $H_{\Omega}(x, y)=G_{\Omega}(x, y)+\frac{1}{2 \pi} \log |x-y|$ be its regular part. We will refer to $G_{\Omega}, H_{\Omega}$ simply as $G$ and $H$ respectively when the dependence in $\Omega$ is not relevant.

First of all, let us point out that if $\inf _{\Omega} V>0$, it is known that if the sequence $u_{\lambda}$ is a family of solutions to (1.1) which is not uniformly bounded from above for $\lambda$ bounded, then $u_{\lambda}$ blows-up at $k$ different points $q_{1}, \ldots, q_{k}$ in $\Omega$ along a sequence $\lambda \rightarrow 8 \pi k$ and $\left(q_{1}, \ldots, q_{k}\right)$ is a critical point for the function:

$$
\mathcal{F}\left(\xi_{1}, \ldots, \xi_{k}\right)=\sum_{i=1}^{k} H_{\Omega}\left(\xi_{i}, \xi_{i}\right)+\sum_{\substack{i, j=1 \\ i \neq j}}^{k} G_{\Omega}\left(\xi_{i}, \xi_{j}\right)+\frac{1}{4 \pi} \sum_{i=1}^{k} \log V\left(\xi_{i}\right)
$$

(cf. $[8,22,28]$; see also [35,38]). Secondly we note that, if $\lambda \in(0,8 \pi)$ problem $(1.1)$ admits a solution $u_{\min , \lambda}$ corresponding to a global minimum point for the functional

$$
J_{\lambda}(u)=\frac{1}{2} \int_{\Omega}|\nabla u|^{2}-\lambda \log \left(\int_{\Omega} V(x) \mathrm{e}^{u}\right), \quad u \in \mathrm{H}_{0}^{1}(\Omega),
$$

which is coercive by the Moser-Trudinger inequality (cf. [32,40]).

Let us consider the case $V(x) \equiv 1$. In $[9,10]$ the authors study the asymptotic behavior of $u_{\min , \lambda}$ as $\lambda \rightarrow 8 \pi^{-}$, and show that either $u_{\min , \lambda}$ converges to a minimum of $J_{8 \pi}$ or admits a single blow-up point $q \in \Omega$, corresponding to a maximum point of the Robin's function $H_{\Omega}(\cdot, \cdot)$. They observe that both possibilities can occur. For instance, if $\Omega$ is a disk or a simply connected domain sufficiently close to a disk, then concentration does occur as $\lambda \rightarrow 8 \pi^{-}$. On the contrary, there are simply connected regions (for instance, rectangles with large ratio between the sides) for which concentration cannot occur, as $\lambda \rightarrow 8 \pi^{-}$. We also mention that Suzuki in [39] proves that the solution of (1.1) is unique when $\lambda \in(0,8 \pi)$ and $\Omega$ is a simply connected domain. If $\lambda \geqslant 8 \pi$ the situation becomes more 
complex. As well known (see [4]), if $\Omega$ is the unit disk and $\lambda \geqslant 8 \pi$, then there are no solutions to (1.1). In [41] the author constructs a sequence of solutions on simply connected domains "blowing-up" at a critical point of the Robin's function (see also [29,30] and [31] for similar results in a more general setting). Non-simply connected domains are considered in [19].

The general case concerning the existence of solutions with multiple blow-up points so far has been treated only by Baraket-Pacard in [5]. They prove that any nondegenerate critical point $\left(q_{1}, \ldots, q_{k}\right)$ of the function $\mathcal{F}$ defined in (1.3) generates a family of solutions $u_{\lambda}$ which blow-up at $q_{1}, \ldots, q_{k}$ as $\lambda \rightarrow 8 \pi k$. In [21] the author extends the previous result by allowing a general weight function $V(x)$. We are aware of only another successful approach to handle multiple peak blow-up solutions which has been introduced by Chen and Lin in [16] for the annulus. A general degree formula for the corresponding Fredholm map can be found in [15].

Perturbative problems with exponential nonlinearities in dimension two seem to be much more difficult to handle (beside [5], see also [11,12] and [36]) in contrast to similar problems in higher dimensions. Baraket-Pacard's method is too demanding in terms of assumptions (the non-degeneracy of the critical point) and functional framework, and pays in return with a very accurate control on the asymptotics of the solutions. Instead, in the spirit of some perturbation methods available in higher dimension (see e.g. [3] and [37]), we propose an alternative approach to the existence of blowing-up solutions by introducing for our problem a perturbation setting in the space $H_{0}^{1}(\Omega)$. This more flexible approach allows to replace the non-degeneracy assumption of Baraket-Pacard, by showing that "stable" (in a suitable sense) critical points of the function $\mathcal{F}$ in (1.3) generate solutions for (1.1) which blow-up at those points. It is important to point out that this "weaker" stability of critical points enables us to construct some domains where a large number of blowing-up solutions exists.

Let us state now the main results of the paper. Recall $\Omega^{\prime}=\{q \in \Omega: V(q)>0\}$ and let $\Delta=\left\{\left(q_{1}, \ldots, q_{k}\right) \in\right.$ $\Omega^{k}: q_{i}=q_{j}$ for some $\left.i \neq j\right\}$.

Theorem 1.1. Assume that $K$ is a stable critical set for $\mathcal{F}$ in $\left(\Omega^{\prime}\right)^{k} \backslash \Delta$ (see (1.3) and Definition 5.1). Then there exists a family of solutions of (1.1) which blow-up at points $q_{1}, \ldots, q_{k}$ with the property $\left(q_{1}, \ldots, q_{k}\right) \in K$, along a sequence $\lambda \rightarrow 8 \pi k$, in the sense that (1.2) holds.

In Section 5 we take $V(x) \equiv 1$ and we exhibit some simply connected domains where many solutions to (1.1) blowing-up at one or more points exist: see Theorems 5.4, 5.5 and Corollary 5.6. This result is in striking contrast with Suzuki's uniqueness result [39] for the case $\lambda<8 \pi$.

As a consequence of Theorem 1.1, changing sign weight functions $V(x)$ generate many solutions as we show in the following result:

Theorem 1.2. Let $\Omega_{1}^{\prime}, \ldots, \Omega_{v}^{\prime}$ be the connected components of $\Omega^{\prime}$ in $\Omega$. Then there exist at least $v$ families of solutions of (1.1) which blow-up at a maximum point $\xi_{i}$ of the function $\mathcal{F}$ in $\Omega_{i}^{\prime}$ for $i=1, \ldots, v$, along a sequence $\lambda \rightarrow 8 \pi$, in the sense that (1.2) holds.

As far as it concerns the existence of solutions blowing-up at a point when $V(x) \equiv 1$, we would like also to quote the following existence result:

Theorem 1.3. Let $V(x) \equiv 1$. Assume that $c$ is a stable critical value for the Robin's function $H_{\Omega}(\cdot, \cdot)$ (see Definition 6.1). Then there exists a family of solutions of (1.1) which blow-up at a point $q$ with the properties $H_{\Omega}(q, q)=c$ and $\nabla H_{\Omega}(q, q)=0$, along a sequence $\lambda \rightarrow 8 \pi$, in the sense that (1.2) holds.

It allows to find solutions blowing-up at critical points of the Robin's function of "saddle-type", which a priori are not stable according to Definition 5.1. In Section 6 we exhibit some domains where this kind of solutions appears: see Theorems 6.2 and 6.3 . 
Let us sketch the main ideas involved in the proof. A crucial role in the construction of our solution is played by the problem:

$$
\left\{\begin{array}{l}
-\Delta U=\rho^{2} \mathrm{e}^{U} \\
\int_{\mathbb{R}^{2}} \mathrm{e}^{U}<+\infty
\end{array} \text { in } \mathbb{R}^{2},\right.
$$

where $\rho \neq 0$ is a fixed parameter. In [14] (see also the Liouville representation formula in [27]) it is shown that all the solutions to (1.4) take the form:

$$
U_{\tau, \xi}(x)=\log \frac{8 \tau^{2}}{\left(\tau^{2} \rho^{2}+|x-\xi|^{2}\right)^{2}}, \quad x \in \mathbb{R}^{2},
$$

for $\tau>0$ and $\xi \in \mathbb{R}^{2}$. Following Bahri's idea (see [3] and also [37]) we look for solutions to (1.1) in the form

$$
u(x)=\sum_{i=1}^{k} P U_{\tau_{\lambda, i}, \xi_{\lambda, i}}(x)+\phi^{\lambda}(x)
$$

for suitable positive parameters $\tau_{\lambda}=\left(\tau_{\lambda, 1}, \ldots, \tau_{\lambda, k}\right)$ and points $\xi_{\lambda}=\left(\xi_{\lambda, 1}, \ldots, \xi_{\lambda, k}\right)$. Here, the remainder term $\phi^{\lambda}$ goes to zero in $\mathrm{H}_{0}^{1}(\Omega)$ as $\lambda$ goes to $8 \pi k$ and $P U_{\tau, \xi}$ denotes the projection of $U_{\tau, \xi}$ into $\mathrm{H}_{0}^{1}(\Omega)$, in other words, $P U_{\tau, \xi}$ is uniquely defined as satisfying:

$$
\begin{cases}-\Delta P U_{\tau, \xi}=-\Delta U_{\tau, \xi}=\rho^{2} \mathrm{e}^{U_{\tau, \xi}} & \text { in } \Omega \\ P U_{\tau, \xi}=0 & \text { on } \partial \Omega\end{cases}
$$

In order to determine $\tau_{\lambda}$ and $\xi_{\lambda}$ in Section 4 we reduce the problem to a finite dimensional one. Although this problem has many similarities to equations with critical growth in smooth domains of $\mathbb{R}^{N}, N \geqslant 3$, our proof displays important differences. First of all, we point out that the parameter $\tau_{\lambda}$ will be a priori prescribed in terms of the point $\xi_{\lambda}$ (see formula (2.6)). An important consequence is that the function $\phi^{\lambda}$ will be found in a space of codimension $2 k$. We recall that the standard procedure in elliptic problems involving the critical Sobolev exponent takes place in spaces of codimension $(N+1) k$. This different choice of the space will require delicate computations in order to establish some invertibility property of the corresponding linearized operator, which plays a crucial role in the finite dimensional reduction. This analysis will be carried out in Section 3. In Section 4 we study the reduced problem and give the expansion of the functional associated to the problem (1.1). We have collected some technical computations in Appendix A, B, C, D.

We learnt that related results to this paper have been proved at the same time, independently and using different arguments by Del Pino, Kowalczyk and Musso (see [17]).

\section{Setting of the problem}

In order to prove the existence of blow-up solutions to (1.1), we will consider the problem:

$$
\begin{cases}-\Delta u=\rho^{2} V(x) \mathrm{e}^{u} & \text { in } \Omega, \\ u=0 & \text { on } \partial \Omega,\end{cases}
$$

and seek solutions such that

$$
\rho^{2} V(x) \mathrm{e}^{u}-8 \pi \sum_{i=1}^{k} \delta_{q_{i}} \quad \text { in the sense of measures in } \bar{\Omega} \text {, as } \rho \rightarrow 0,
$$

for some point $q=\left(q_{1}, \ldots, q_{k}\right) \in\left(\Omega^{\prime}\right)^{k} \backslash \Delta$. Via the transformation $\rho^{2}=\lambda /\left(\int_{\Omega} V(x) \mathrm{e}^{u}\right)$ it is possible to show that for concentrating solutions problem (2.1) is equivalent to (1.1).

It will be useful to rewrite problem (2.1) in a more convenient setting. For this purpose, let us introduce the following definition. 
Definition 2.1. For $p>1$, let $i_{p}^{*}: \mathrm{L}^{p} \rightarrow \mathrm{H}_{0}^{1}(\Omega)$ be the adjoint operator relative to the immersion $i: \mathrm{H}_{0}^{1}(\Omega) \hookrightarrow$ $\mathrm{L}^{\frac{p}{p-1}}(\Omega)$ and let $i^{*}: \bigcup_{p>1} \mathrm{~L}^{p} \rightarrow \mathrm{H}_{0}^{1}(\Omega)$ be defined by the property $\left.i^{*}\right|_{\mathrm{L}^{p}}=i_{p}^{*}$ for any $p>1$.

By definition, $v=i^{*}(u)$ holds if and only if

$$
(v, \varphi)=\int_{\Omega} u \varphi \mathrm{d} x, \quad \forall \varphi \in \mathrm{H}_{0}^{1}(\Omega),
$$

and the following estimate holds:

Lemma 2.2. For any $p>1$ there exists $c_{p}>0$ such that

$$
\left\|i^{*}(u)\right\| \leqslant c_{p}\|u\|_{\mathrm{L}}^{p}, \quad \forall u \in \mathrm{H}_{0}^{1}(\Omega) .
$$

$\mathrm{H}_{0}^{1}(\Omega)$ is an Hilbert space equipped with the usual inner product

$$
(u, v)=\int_{\Omega} \nabla u \nabla v \mathrm{~d} x
$$

and induced norm

$$
\|u\|=\left(\int_{\Omega}|\nabla u|^{2} \mathrm{~d} x\right)^{1 / 2} .
$$

Next, we recall the Moser-Trudinger inequality (cf. $[32,40])$ :

Lemma 2.3. There exists a constant $c>0$ such that for any smooth bounded domain $\Omega \subset \mathbb{R}^{2}$ we have:

$$
\int_{\Omega} \mathrm{e}^{4 \pi u^{2} /\|u\|_{\mathrm{H}_{0}^{1}}^{2}} \leqslant c|\Omega|, \quad \forall u \in \mathrm{H}_{0}^{1}(\Omega) .
$$

In particular, by Lemma 2.3 we deduce the following useful estimate:

Remark 2.4. There exists a constant $c>0$ such that for any $\eta>0$,

$$
\int_{\Omega} \mathrm{e}^{\eta u} \leqslant c|\Omega| \mathrm{e}^{\frac{\eta^{2}}{16 \pi}\|u\|_{\mathrm{H}_{0}^{1}}^{2}}, \quad \forall u \in \mathrm{H}_{0}^{1}(\Omega) .
$$

In particular, the map:

$$
\begin{aligned}
\mathrm{H}_{0}^{1}(\Omega) & \rightarrow L^{p}(\Omega), \\
u & \rightarrow \mathrm{e}^{u}
\end{aligned}
$$

is continuous for every $p>1$ and we can rewrite problem (2.1) in the following equivalent form:

$$
\left\{\begin{array}{l}
u=i^{*}\left(\rho^{2} V(x) \mathrm{e}^{u}\right) \quad \text { in } \Omega, \\
u \in \mathrm{H}_{0}^{1}(\Omega) .
\end{array}\right.
$$

Let $P U_{\tau, \xi}$ be the projection onto $\mathrm{H}_{0}^{1}(\Omega)$ of $U_{\tau, \xi}$ (see (1.5), (1.6)). Thus, by Definition 2.1 we have

$$
P U_{\tau, \xi}:=i^{*}\left(\rho^{2} \mathrm{e}^{U_{\tau, \xi}}\right) .
$$


We are looking for solutions to (2.2) of the form

$$
u(x)=\sum_{i=1}^{k} P U_{\tau_{\rho, i}, \xi_{\rho, i}}(x)+\phi^{\rho}(x)
$$

for suitable positive parameters $\tau_{\rho}=\left(\tau_{\rho, 1}, \ldots, \tau_{\rho, 1}\right)$ and points $\xi_{\rho}=\left(\xi_{\rho, 1}, \ldots, \xi_{\rho, k}\right)$. The remainder term $\phi^{\rho}$ belongs to a suitable space which will be defined as follows. Let

$$
\psi_{\tau, \xi}^{0}(x)=\frac{\partial U_{\tau, \xi}}{\partial \tau}(x)=\frac{2}{\tau} \frac{|x-\xi|^{2}-\tau^{2} \rho^{2}}{|x-\xi|^{2}+\tau^{2} \rho^{2}}, \quad x \in \Omega,
$$

and for $j=1,2$ let

$$
\psi_{\tau, \xi}^{j}(x)=\frac{\partial U_{\tau, \xi}}{\partial \xi_{j}}(x)=4 \frac{(x-\xi)_{j}}{\tau^{2} \rho^{2}+|x-\xi|^{2}}, \quad x \in \Omega
$$

For $j=0,1,2$, the function $\psi_{\tau, \xi}^{j}$ is a solution for the equation $-\Delta \psi=\rho^{2} \mathrm{e}^{U_{\tau, \xi}} \psi$ in $\mathbb{R}^{2}$. Let $P \psi_{\tau, \xi}^{j}$ be the projection into $\mathrm{H}_{0}^{1}(\Omega)$ of $\psi_{\tau, \xi}^{j}$, i.e.

$$
\begin{cases}-\Delta P \psi_{\tau, \xi}^{j}=\rho^{2} \mathrm{e}^{U_{\tau, \xi}} \psi_{\tau, \xi}^{j} & \text { in } \Omega, \\ P \psi_{\tau, \xi}^{j}=0 & \text { on } \partial \Omega .\end{cases}
$$

For any $\xi=\left(\xi_{1}, \ldots, \xi_{k}\right) \in\left(\Omega^{\prime}\right)^{k} \backslash \Delta$, set

$$
\tau_{i}(\xi)=\sqrt{\frac{V\left(\xi_{i}\right)}{8}} \mathrm{e}^{4 \pi\left(H_{\Omega}\left(\xi_{i}, \xi_{i}\right)+\sum_{j \neq i} G_{\Omega}\left(\xi_{i}, \xi_{j}\right)\right)} .
$$

Set $U_{i}=U_{\tau_{i}(\xi), \xi_{i}}$ and $\psi_{i}^{j}:=\psi_{\tau_{i}(\xi), \xi_{i}}^{j}$ for $j=0,1,2, i=1, \ldots, k$ and $\xi \in\left(\Omega^{\prime}\right)^{k} \backslash \Delta$. We consider the subspace of $\mathrm{H}_{0}^{1}(\Omega)$ :

$$
K_{\xi}=\operatorname{span}\left\{P \psi_{i}^{j}, j=1,2, i=1, \ldots, k\right\},
$$

and its complement

$$
K_{\xi}^{\perp}=\left\{\phi \in \mathrm{H}_{0}^{1}(\Omega) \mid\left(\phi, P \psi_{i}^{j}\right)=0, j=1,2, i=1, \ldots, k\right\},
$$

and consider the corresponding orthogonal projections:

$$
\Pi_{\xi}: \mathrm{H}_{0}^{1}(\Omega) \rightarrow K_{\xi} \quad \text { and } \quad \Pi_{\xi}^{\perp}: \mathrm{H}_{0}^{1}(\Omega) \rightarrow K_{\xi}^{\perp} .
$$

\section{The linear problem: a key lemma}

Let us introduce the linear operator $L_{\xi}^{\rho}: K_{\xi}^{\perp} \rightarrow K_{\xi}^{\perp}$ defined as follows:

$$
L_{\xi}^{\rho}(\phi)=\Pi_{\xi}^{\perp}\left\{\phi-i^{*}\left[\rho^{2} V(x) \mathrm{e}^{\sum_{i=1}^{k} P U_{i}} \phi\right]\right\} .
$$

In order to solve Eq. (4.1), a crucial ingredient is given by the following result, which is similar in spirit to an invertibility property established in [11]:

Proposition 3.1. Let $\xi \in\left(\Omega^{\prime}\right)^{k} \backslash \Delta$. There exists $\rho_{0}>0$ and a constant $c>0$ such that for any $\rho \in\left(0, \rho_{0}\right)$ we have

$$
\left\|L_{\xi}^{\rho}(\phi)\right\| \geqslant \frac{c}{|\log \rho|}\|\phi\|, \quad \forall \phi \in K_{\xi}^{\perp} .
$$

In particular, the operator $L_{\xi}^{\rho}$ is invertible and $\left\|\left(L_{\xi}^{\rho}\right)^{-1}\right\| \leqslant|\log \rho| / c$.

Moreover, the estimates are uniform in compact sets of $\left(\Omega^{\prime}\right)^{k} \backslash \Delta$. 
Proof. We need only to establish the validity of the following estimate:

$$
\left\|L_{\xi}^{\rho}(\phi)\right\| \geqslant \frac{c}{|\log \rho|}\|\phi\| \quad \forall \phi \in K_{\xi}^{\perp},
$$

for some uniform constant $c>0$. Indeed, by (3.1) we deduce that $L_{\xi}^{\rho}$ is an injective operator with closed image. Since $L_{\xi}^{\rho}$ is selfadjoint, we also conclude that it is onto and its inverse $\left(L_{\xi}^{\rho}\right)^{-1}$ satisfies $\left\|\left(L_{\xi}^{\rho}\right)^{-1}\right\| \leqslant|\log \rho| / c$.

To prove (3.1), we argue by contradiction. Suppose there exist sequences

$$
\rho \rightarrow 0, \xi \rightarrow \xi_{0} \in\left(\Omega^{\prime}\right)^{k} \backslash \Delta, \phi \in K_{\xi}^{\perp}:\|\phi\|=1 \text { and }\left\|L_{\xi}^{\rho}(\phi)\right\|=o\left(\frac{1}{|\log \rho|}\right) .
$$

Write,

$$
\phi-i^{*}\left[\rho^{2} V(x) \mathrm{e}^{\sum_{i=1}^{k} P U_{i}} \phi\right]=\psi+w,
$$

where $\psi \in K_{\xi}^{\perp}, w \in K_{\xi}$ and $\|\psi\|=o(1 /|\log \rho|) \rightarrow 0$. Equivalently (in a weak sense), there holds

$$
\begin{cases}-\Delta \phi=\rho^{2} V(x) \mathrm{e}^{\sum_{i=1}^{k} P U_{i}} \phi-\Delta(\psi+w) & \text { in } \Omega, \\ \phi=0 & \text { on } \partial \Omega .\end{cases}
$$

Step 1. For any $p \in(1,2)$,

$$
\|w\|=O\left(\rho^{(2-p) / p}\right) \rightarrow 0 .
$$

Let $w=\sum_{h=1}^{k} \sum_{l=1}^{2} c_{h l} P \psi_{h}^{l}$. We multiply (3.3) by $P \psi_{s}^{j}, j=1,2$ and $s=1, \ldots, k$, and get

$$
\sum_{h=1}^{k} \sum_{l=1}^{2} c_{h l}\left(P \psi_{h}^{l}, P \psi_{s}^{j}\right)_{\mathrm{H}_{0}^{1}}=-\rho^{2} \int_{\Omega} V(x) \mathrm{e}^{\sum_{i=1}^{k} P U_{i}} \phi P \psi_{s}^{j} .
$$

By Lemma A.4 the L.H.S. of (3.5) is estimated as follows:

$$
\text { L.H.S. }=\frac{D}{\tau^{2} \rho^{2}} c_{s j}+O\left(\sum_{h=1}^{k} \sum_{l=1}^{2}\left|c_{h l}\right|\right) .
$$

Moreover, the R.H.S. of (3.5) takes the form:

$$
\begin{aligned}
& -\rho^{2} \int_{\Omega} V(x) \mathrm{e}^{\sum_{i=1}^{k} P U_{i}} \phi P \psi_{s}^{j} \\
& =\int_{\Omega}\left[\rho^{2} \sum_{i=1}^{k} \mathrm{e}^{U_{i}}-\rho^{2} V(x) \mathrm{e}^{\sum_{i=1}^{k} P U_{i}}\right] \phi P \psi_{s}^{j}-\rho^{2} \int_{\Omega} \mathrm{e}^{U_{s}} \phi\left[P \psi_{s}^{j}-\psi_{s}^{j}\right]-\rho^{2} \sum_{i \neq s} \int_{\Omega} \mathrm{e}^{U_{i}} \phi P \psi_{s}^{j},
\end{aligned}
$$

where we have used (2.5) and $\phi \in K_{\xi}^{\perp}$. Fix $p \in(1,2)$ and use Lemmata A.4 and B.1, together with Hölder's inequality (here $1 / q+1 / p<1$ ), to get

$$
\begin{aligned}
& \left|\int_{\Omega}\left[\rho^{2} V(x) \mathrm{e}^{\sum_{i=1}^{k} P U_{i}}-\rho^{2} \sum_{i=1}^{k} \mathrm{e}^{U_{i}}\right] \phi P \psi_{s}^{j}\right| \\
& \quad \leqslant\left\|\rho^{2} V(x) \mathrm{e}^{\sum_{i=1}^{k} P U_{i}}-\rho^{2} \sum_{i=1}^{k} \mathrm{e}^{U_{i}}\right\|_{\mathrm{L}^{p}}\|\phi\|_{\mathrm{L} q}\left\|P \psi_{s}^{j}\right\|=O\left(\rho^{2(1-p) / p}\right) .
\end{aligned}
$$


Using Lemma A.2, Proposition A.3 and Hölder's inequality (with $1 / q+1 / p=1$ ), we get,

$$
\left|\rho^{2} \int_{\Omega} \mathrm{e}^{U_{s}} \phi\left[P \psi_{s}^{j}-\psi_{s}^{j}\right]\right|=O\left(\left\|\rho^{2} \mathrm{e}^{U_{s}}\right\|_{\mathrm{L}^{p}}\|\phi\|_{\mathrm{L}^{q}}\right)=O\left(\rho^{2(1-p) / p}\right) .
$$

Using Lemma C.6 and Hölder's inequality (with $1 / q+1 / p=1$ ), we obtain,

$$
\left|\rho^{2} \sum_{i \neq s} \int_{\Omega} \mathrm{e}^{U_{i}} \phi P \psi_{s}^{j}\right|=O\left(\sum_{i \neq s}\left\|\rho^{2} \mathrm{e}^{U_{i}} P \psi_{s}^{j}\right\|_{\mathrm{L}^{p}}\|\phi\|_{\mathrm{L}^{q}}\right)=O\left(\rho^{2 / p}\right) .
$$

Then, by (3.7)-(3.10) it follows that the R.H.S. of (3.5) satisfies:

$$
\text { R.H.S. }=O\left(\rho^{2(1-p) / p}\right) \text {. }
$$

Inserting the estimates (3.6) and (3.11) into (3.5), we deduce that

$$
\sum_{h=1}^{k} \sum_{l=1}^{2}\left|c_{h l}\right|=O\left(\rho^{2 / p}\right)
$$

Finally, by Lemma A.4 and by (3.12) we deduce that

$$
\|w\|=O\left(\rho^{(2-p) / p}\right) \rightarrow 0
$$

and claim (3.4) is proved.

Step 2. For any $i=1, \ldots, k$

$$
\left(\phi, P \psi_{i}^{0}\right)_{\mathrm{H}_{0}^{1}} \rightarrow 0 \text {. }
$$

Let $i=1, \ldots, k$. Note that, for $\tau_{i}>0$ and $\xi_{i} \in \Omega$, the functions

$$
w_{i}(x)=\frac{4}{3 \tau_{i}} \log \left(\tau_{i}^{2} \rho^{2}+\left|x-\xi_{i}\right|^{2}\right) \frac{\tau_{i}^{2} \rho^{2}-\left|x-\xi_{i}\right|^{2}}{\tau_{i}^{2} \rho^{2}+\left|x-\xi_{i}\right|^{2}}+\frac{8}{3} \frac{\tau_{i} \rho^{2}}{\tau_{i}^{2} \rho^{2}+\left|x-\xi_{i}\right|^{2}}
$$

and

$$
t_{i}(x)=-2 \frac{\tau_{i} \rho^{2}}{\tau_{i}^{2} \rho^{2}+\left|x-\xi_{i}\right|^{2}}
$$

satisfy: $-\Delta w_{i}-\rho^{2} \mathrm{e}^{U_{\tau_{i}, \xi_{i}}} w_{i}=\rho^{2} \mathrm{e}^{U_{\tau_{i}, \xi_{i}}} \psi_{\tau_{i}, \xi_{i}}^{0}$ and $-\Delta t_{i}-\rho^{2} \mathrm{e}^{U_{\tau_{i}, \xi_{i}} t_{i}}=\rho^{2} \mathrm{e}^{U_{\tau_{i}, \xi_{i}}}$ in $\mathbb{R}^{2}$ respectively. A straightforward calculation shows that,

$$
\int_{\Omega}\left|\nabla w_{i}\right|^{2}=M_{i}^{2}(1+o(1))(\log \rho)^{2}, \quad \int_{\Omega}\left|\nabla t_{i}\right|^{2}=O(1) \quad \text { as } \rho \rightarrow 0
$$

with $M_{i}=\frac{32}{3 \tau_{i}}\left(\int_{\mathbb{R}^{2}} \frac{|y|^{2}}{\left(1+|y|^{2}\right)^{4}}\right)^{1 / 2}$, and (3.14) holds uniformly in $\operatorname{dist}\left(\xi_{i}, \partial \Omega\right) \geqslant \varepsilon, \varepsilon>0$, and $\tau_{i}$ bounded away from zero.

Let now $\tau_{i}=\tau_{i}(\xi)$ and $\xi$ as specified in (3.2). The projection $P u_{i} \in \mathrm{H}_{0}^{1}(\Omega)$ of the function

$$
u_{i}=w_{i}+\frac{16 \pi}{3 \tau_{i}} H\left(\xi_{i}, \xi_{i}\right) t_{i}
$$

satisfies

$$
-\Delta P u_{i}-\rho^{2} V(x) \mathrm{e}^{\sum_{h=1}^{k} P U_{h}} P u_{i}=\rho^{2} \mathrm{e}^{U_{i}} \psi_{i}^{0}-\rho^{2} \sum_{h \neq i} \mathrm{e}^{U_{h}} \frac{16 \pi}{3 \tau_{i}} G\left(\xi_{h}, \xi_{i}\right)+F_{i},
$$


where

$$
\begin{aligned}
F_{i}(x)= & -\frac{16 \pi}{3 \tau_{i}}\left(H\left(x, \xi_{i}\right)-H\left(\xi_{i}, \xi_{i}\right)\right) \rho^{2} \mathrm{e}^{U_{i}}+\left(u_{i}-P u_{i}+\frac{16 \pi}{3 \tau_{i}} H\left(x, \xi_{i}\right)\right) \rho^{2} \mathrm{e}^{U_{i}} \\
& +\left(\rho^{2} \sum_{h=1}^{k} \mathrm{e}^{U_{h}}-\rho^{2} V(x) \mathrm{e}^{\sum_{h=1}^{k} P U_{h}}\right) P u_{i}+\rho^{2} \sum_{h \neq i} \mathrm{e}^{U_{h}}\left(\frac{16 \pi}{3 \tau_{i}} G\left(\xi_{h}, \xi_{i}\right)-P u_{i}\right) .
\end{aligned}
$$

Since $u_{i}-P u_{i}+\frac{16 \pi}{3 \tau_{i}} H\left(x, \xi_{i}\right)$ is an harmonic function with boundary values satisfying

$$
\left\|u_{i}-P u_{i}+\frac{16 \pi}{3 \tau_{i}} H\left(x, \xi_{i}\right)\right\|_{2, \alpha, \partial \Omega} \leqslant C \rho^{2}
$$

for any $\alpha \in(0,1)$, by elliptic regularity theory it follows that

$$
\left\|u_{i}-P u_{i}+\frac{16 \pi}{3 \tau_{i}} H\left(x, \xi_{i}\right)\right\|_{2, \alpha, \Omega} \leqslant C \rho^{2}
$$

for any $\alpha \in(0,1)$. Hence, we get that

$$
\begin{aligned}
& P u_{i}(x)=\frac{16 \pi}{3 \tau_{i}} G\left(x, \xi_{i}\right)+O\left(\rho^{2}\right) \text { in } C_{\mathrm{loc}}^{0}\left(\bar{\Omega} \backslash\left\{\xi_{i}\right\}\right), \\
& \left\|P u_{i}\right\|=M_{i}(1+o(1))|\log \rho|,
\end{aligned}
$$

and by means of Lemmata A.2, B.1, from (3.15) we deduce that $f_{i}=i^{*}\left(F_{i}\right)$ satisfies:

$$
\left\|f_{i}\right\|=o(1), \quad \text { as } \rho \rightarrow 0 .
$$

Therefore,

$$
P u_{i}-i^{*}\left(\rho^{2} V(x) \mathrm{e}^{\sum_{h=1}^{k} P U_{h}} P u_{i}\right)=P \psi_{i}^{0}-\frac{16 \pi}{3 \tau_{i}} \sum_{h \neq i} G\left(\xi_{h}, \xi_{i}\right) P U_{h}+f_{i}
$$

with $\left\|f_{i}\right\| \rightarrow 0$. Multiply (3.3) by $P u_{i}$ and use (3.18) to obtain:

$$
\begin{aligned}
\left(\phi, P \psi_{i}^{0}\right)_{\mathrm{H}_{0}^{1}} & =\frac{16 \pi}{3 \tau_{i}} \sum_{h \neq i} G\left(\xi_{h}, \xi_{i}\right)\left(\phi, P U_{h}\right)_{\mathrm{H}_{0}^{1}}+o\left(\frac{\left\|P u_{i}\right\|}{|\log \rho|}\right)+o(1) \\
& =\frac{16 \pi}{3 \tau_{i}} \sum_{h \neq i} G\left(\xi_{h}, \xi_{i}\right)\left(\phi, P U_{h}\right)_{\mathrm{H}_{0}^{1}}+o(1)
\end{aligned}
$$

where we have taken in account (3.2), (3.17) and the estimate in Step 1. In conclusion, we need to show that $\left(\phi, P U_{h}\right)_{\mathrm{H}_{0}^{1}}=o(1)$ for any $h=1, \ldots, k$. Multiply (3.3) by $P \psi_{i}^{0}$ and use Lemmata A.4, B.1 to obtain:

$$
\rho^{2} \int_{\Omega} \mathrm{e}^{U_{i}} \phi\left(\psi_{i}^{0}-P \psi_{i}^{0}\right)=\rho^{2} \sum_{h \neq i} \int_{\Omega} \mathrm{e}^{U_{h}} \phi P \psi_{i}^{0}+o(1) .
$$

By (A.3), (A.4) and Lemmata A.2, A.4, finally we deduce that

$$
\left(\phi, P U_{i}\right)_{\mathrm{H}_{0}^{1}}=\rho^{2} \int_{\Omega} \mathrm{e}^{U_{i}} \phi=o(1),
$$

and (3.13) is completely established.

Define the spaces 


$$
\begin{aligned}
& \mathrm{L}:=\left\{\Phi:\left\|\frac{\Phi}{1+|y|^{2}}\right\|_{\mathrm{L}^{2}\left(\mathbb{R}^{2}\right)}<+\infty\right\}, \\
& \mathrm{H}:=\left\{\Phi:\|\nabla \Phi\|_{\mathrm{L}^{2}\left(\mathbb{R}^{2}\right)}+\left\|\frac{\Phi}{1+|y|^{2}}\right\|_{\mathrm{L}^{2}\left(\mathbb{R}^{2}\right)}<+\infty\right\}
\end{aligned}
$$

endowed respectively with the norm $\|\Phi\|_{\mathrm{L}}=\left\|\Phi /\left(1+|y|^{2}\right)\right\|_{\mathrm{L}^{2}\left(\mathbb{R}^{2}\right)}$ and $\|\Phi\|_{\mathrm{H}}=\left(\|\nabla \Phi\|_{\mathrm{L}^{2}\left(\mathbb{R}^{2}\right)}^{2}+\|\Phi\|_{\mathrm{L}}^{2}\right)^{1 / 2}$. If $S^{2}$ denotes the unit sphere in $\mathbb{R}^{3}$ with the standard metric and $\pi_{N}$ is the stereographic projection through the north pole, let us point out that the map $\Phi \rightarrow \Phi \circ \pi_{N}$ is an isometry from $\mathrm{L}$ to $L^{2}\left(S^{2}\right)$ and from $\mathrm{H}$ to $H^{1}\left(S^{2}\right)$. Hence, by the compactness of the embedding $H^{1}\left(S^{2}\right) \hookrightarrow L^{2}\left(S^{2}\right)$, we get the compactness of the embedding $\mathrm{H} \hookrightarrow \mathrm{L}$.

Assume that $\xi \in \mathcal{O}_{\varepsilon}$ for some $\varepsilon>0$, where

$$
\mathcal{O}_{\varepsilon}=\left\{\left(q_{1}, \ldots, q_{k}\right) \in \Omega^{k}: \operatorname{dist}\left(q_{i}, \partial \Omega\right) \geqslant 2 \varepsilon,\left|q_{i}-q_{j}\right| \geqslant 2 \varepsilon \text { for } i \neq j\right\} .
$$

Let $\chi: \mathbb{R} \rightarrow[0,1]$ be a smooth cut-off function such that $\chi(x)=1$ if $|x| \leqslant \varepsilon / 2, \chi(x)=0$ if $|x| \geqslant \varepsilon$. For any $i=1, \ldots, k$ set

$$
\tilde{\phi}^{i}(y)=\phi^{i}(y) \chi^{i}(y), \quad y \in \Omega^{i}:=\frac{\Omega-\xi_{i}}{\tau_{i} \rho},
$$

where $\phi^{i}(y)=\phi\left(\tau_{i} \rho y+\xi_{i}\right)$ and $\chi^{i}(y)=\chi\left(\tau_{i} \rho y\right)$. We will always consider $\tilde{\phi}^{i}$ extended to be zero outside $\Omega^{i}$.

Step 3. For any $i=1, \ldots, k$

$$
\tilde{\phi}^{i} \rightarrow 0 \text { weakly in } \mathrm{H} \text {. }
$$

First of all, we remark that the function $\tilde{\phi}^{i}$ satisfies (in a weak sense),

$$
\begin{cases}-\Delta \tilde{\phi}^{i}=a^{i}(y) \tilde{\phi}^{i}-\Delta \tilde{z}^{i}+\tilde{v}^{i} & \text { in } \Omega^{i}, \\ \tilde{\phi}^{i}=0 & \text { on } \partial \Omega^{i},\end{cases}
$$

where

$$
\begin{aligned}
& a^{i}(y)=\tau_{i}^{2} \rho^{4} V\left(\tau_{i} \rho y+\xi_{i}\right) \mathrm{e}^{\sum_{h=1}^{k} P U_{h}\left(\tau_{i} \rho y+\xi_{i}\right)}, \\
& \tilde{z}^{i}(y)=z^{i}(y) \chi^{i}(y), \quad z^{i}(y)=(\psi+w)\left(\tau_{i} \rho y+\xi_{i}\right), \\
& \tilde{v}^{i}(y)=-\Delta \chi^{i}(y)\left(\phi^{i}-z^{i}\right)-2 \nabla \chi^{i}(y) \nabla\left(\phi^{i}-z^{i}\right) .
\end{aligned}
$$

Observe that by Lemma B.1 we have,

$$
\begin{aligned}
\rho^{2} \sum_{h=1}^{k} \int_{\Omega} \mathrm{e}^{U_{h}} \phi^{2} & =\rho^{2} \int_{\Omega} V(x) \mathrm{e}^{\sum_{h=1}^{k} P U_{h}} \phi^{2}+O\left(\int_{\Omega}\left|\rho^{2} \sum_{h=1}^{k} \mathrm{e}^{U_{h}}-\rho^{2} V(x) \mathrm{e}^{\sum_{h=1}^{k} P U_{h}}\right| \phi^{2}\right) \\
& =\rho^{2} \int_{\Omega} V(x) \mathrm{e}^{\sum_{h=1}^{k} P U_{h}} \phi^{2}+o(1) .
\end{aligned}
$$

Multiplying (3.3) by $\phi$, we obtain that

$$
\rho^{2} \int_{\Omega} V(x) \mathrm{e}^{\sum_{h=1}^{k} P U_{h}} \phi^{2}=\int_{\Omega}|\nabla \phi|^{2}-\int_{\Omega} \nabla \psi \nabla \phi=O(1) .
$$

By (3.21), (3.22) we get that $\tilde{\phi}^{i}$ is bounded in $\mathrm{H}$, since

$$
\int_{\Omega^{i}}\left|\nabla \tilde{\phi}^{i}\right|^{2}=O\left(\int_{\Omega}|\nabla \phi|^{2}\right)=O(1)
$$


and

$$
\int_{\Omega^{i}} \frac{\left(\tilde{\phi}^{i}(y)\right)^{2}}{\left(1+|y|^{2}\right)^{2}} \mathrm{~d} y=\frac{\rho^{2}}{8} \int_{\Omega} \mathrm{e}^{U_{i}} \phi^{2} \chi^{2}\left(x-\xi_{i}\right)=O(1) .
$$

Hence, we can assume (up to a subsequence) that $\tilde{\phi}^{i} \rightarrow \phi_{0}^{i}$ in $\mathrm{H}$ and strongly in L. On the other hand, by Proposition A.1 it follows that

$$
a^{i}(y)=\frac{V\left(\tau_{i} \rho y+\xi_{i}\right)}{\tau_{i}^{2}\left(1+|y|^{2}\right)^{2}} \mathrm{e}^{8 \pi\left(H\left(\tau_{i} \rho y+\xi_{i}, \xi_{i}\right)+\sum_{h \neq i} G\left(\tau_{i} \rho y+\xi_{i}, \xi_{h}\right)\right)+O\left(\rho^{2}\right)} \rightarrow \frac{8}{\left(1+|y|^{2}\right)^{2}}
$$

uniformly on compact sets of $\mathbb{R}^{2}$. Furthermore,

$$
\left\|\nabla \tilde{z}^{i}\right\|_{L^{2}\left(\mathbb{R}^{2}\right)}^{2}=O\left(\int_{\Omega}|\nabla \psi|^{2}+|\nabla w|^{2}\right) \rightarrow 0
$$

and, for any $\Psi \in C_{0}^{\infty}\left(\mathbb{R}^{2}\right)$ we have,

$$
\int_{\mathbb{R}^{2}} \tilde{v}^{i} \Psi=\int_{\Omega}\left[-\Delta \chi\left(x-\xi_{i}\right)(\phi-\psi-w)(x)-2 \nabla \chi\left(x-\xi_{i}\right) \nabla(\phi-\psi-w)(x)\right] \Psi\left(\frac{x-\xi_{i}}{\tau_{i} \rho}\right) \mathrm{d} x=0
$$

if $\varepsilon /\left(2 \tau_{i} \rho\right) \geqslant \operatorname{dist}(\operatorname{Supp} \Psi, 0)$. Finally, for $j=1,2$ we see that,

$$
\int_{\mathbb{R}^{2}} \frac{|y|^{2}-1}{\left(|y|^{2}+1\right)^{3}} \phi_{0}^{i} \mathrm{~d} y=\int_{\mathbb{R}^{2}} \frac{y_{j}}{\left(|y|^{2}+1\right)^{3}} \phi_{0}^{i} \mathrm{~d} y=0 .
$$

Indeed,

$$
\frac{16}{\tau_{i}} \int_{\mathbb{R}^{2}} \frac{|y|^{2}-1}{\left(1+|y|^{2}\right)^{3}} \tilde{\phi}^{i} \mathrm{~d} y=\rho^{2} \int_{\Omega} \mathrm{e}^{U_{i}} \psi_{i}^{0} \phi \chi\left(x-\xi_{i}\right)=\left(\phi, P \psi_{i}^{0}\right)_{\mathrm{H}_{0}^{1}}+O\left(\rho^{2}\right) \rightarrow 0
$$

(by Step 2), and the orthogonality conditions give,

$$
32 \int_{\mathbb{R}^{2}} \frac{y_{j}}{\left(1+|y|^{2}\right)^{3}} \tilde{\phi}^{i} \mathrm{~d} y=\tau_{i} \rho^{3} \int_{\Omega} \mathrm{e}^{U_{i}} \psi_{i}^{j} \phi \chi\left(x-\xi_{i}\right)=O\left(\rho^{3}\right) \rightarrow 0, \quad j=1,2,
$$

and so (3.26) follows by observing that $\tilde{\phi}^{i} /\left(1+|y|^{2}\right) \rightarrow \phi_{0}^{i} /\left(1+|y|^{2}\right)$ in $\mathrm{L}^{2}\left(\mathbb{R}^{2}\right)$. In conclusion, by (3.20)-(3.23), (3.25) we obtain that $\phi_{0}^{i} \in H$ is a (distributional) solution for

$$
-\Delta \phi_{0}^{i}=\frac{8}{\left(1+|y|^{2}\right)^{2}} \phi_{0}^{i} \quad \text { in } \mathbb{R}^{2}
$$

satisfying:

$$
\int_{\mathbb{R}^{2}} \frac{|y|^{2}-1}{\left(|y|^{2}+1\right)^{3}} \phi_{0}^{i} \mathrm{~d} y=\int_{\mathbb{R}^{2}} \frac{y_{j}}{\left(|y|^{2}+1\right)^{3}} \phi_{0}^{i} \mathrm{~d} y=0 \quad \text { for } j=1,2 .
$$

The isometry between $H$ and $H^{1}\left(S^{2}\right)$ and elliptic regularity theory (see [24]) imply that $\phi_{0}^{i}$ is actually a regular solution of (3.27). By Lemma D.1 we get that necessarily $\phi_{0}^{i}=0$ and (3.19) is established.

Step 4. A contradiction arises!

By the compactness of the embedding $L \hookrightarrow H$, we have that $\tilde{\phi}^{i} \rightarrow 0$ in $\mathrm{L}$ for any $i=1, \ldots, k$. By (3.21) and $\|\psi\|=o(1)$ we have, 


$$
\begin{aligned}
\rho^{2} \int_{\Omega} V(x) \mathrm{e}^{\sum_{i=1}^{k} P U_{i}} \phi^{2}+\int_{\Omega} \nabla \psi \nabla \phi & =\rho^{2} \sum_{i=1}^{k} \int_{\Omega} \mathrm{e}^{U_{i}} \phi^{2} \chi^{2}\left(x-\xi_{i}\right)+o(1) \\
& =\sum_{i=1}^{k} \int_{\mathbb{R}^{2}} \frac{8}{\left(1+|y|^{2}\right)^{2}}\left(\tilde{\phi}^{i}\right)^{2} \mathrm{~d} y+o(1) \rightarrow 0,
\end{aligned}
$$

in contradiction with the relation:

$$
1=\int_{\Omega}|\nabla \phi|^{2}=\rho^{2} \int_{\Omega} V(x) \mathrm{e}^{\sum_{i=1}^{k} P U_{i}} \phi^{2}+\int_{\Omega} \nabla \psi \nabla \phi .
$$

This completes the proof of Proposition 3.1.

Remark 3.2. Let us remark that the result in Proposition 3.1 is "sharp" in the sense that in [22] it is shown that for $k=1$ we have also $\left\|\left(L_{\xi}^{\rho}\right)^{-1}\right\| \geqslant c_{1}|\log \rho|$ for some positive constant $c_{1}<c$.

\section{The reduced problem and the functional expansion}

Our first goal will be to prove that, for any $\rho>0$ small enough and for any point $\xi \in\left(\Omega^{\prime}\right)^{k} \backslash \Delta$, there exists $\phi_{\xi}^{\rho} \in K_{\xi}^{\perp}$ such that

$$
\Pi_{\xi}^{\perp}\left[\sum_{i=1}^{k} P U_{i}+\phi_{\xi}^{\rho}-i^{*}\left(\rho^{2} V(x) \mathrm{e}^{\sum_{i=1}^{k} P U_{i}+\phi_{\xi}^{\rho}}\right)\right]=0 .
$$

Proposition 4.1. Let $\xi=\left(\xi_{1}, \ldots, \xi_{k}\right)$ in a compact set of $\left(\Omega^{\prime}\right)^{k} \backslash \Delta$ and $\tau_{i}=\tau_{i}(\xi)$ be given in (2.6). For any $p \in\left(1, \frac{4}{3}\right.$ ) there exists $\rho_{0}>0$ and $R>0$ (uniformly in $\xi$ ) such that for any $\rho \in\left(0, \rho_{0}\right)$ there exists a unique $\phi_{\xi}^{\rho} \in K_{\xi}^{\perp}$ such that

$$
\Pi_{\xi}^{\perp}\left[\sum_{i=1}^{k} P U_{i}+\phi_{\xi}^{\rho}-i^{*}\left(\rho^{2} V(x) \mathrm{e}^{\sum_{i=1}^{k} P U_{i}+\phi_{\xi}^{\rho}}\right)\right]=0
$$

and

$$
\left\|\phi_{\xi}^{\rho}\right\| \leqslant R \rho^{(2-p) / p}|\log \rho|
$$

Proof. According to Proposition 3.1, for $\rho$ small $\left(L_{\xi}^{\rho}\right)^{-1}$ is a linear operator from $K_{\xi}^{\perp}$ into itself such that $\left\|\left(L_{\xi}^{\rho}\right)^{-1}\right\| \leqslant c_{2}|\log \rho|$ uniformly in $\xi$. Let us point out that $\phi$ is a solution of (4.2) if and only if it is a fixed point of the operator $T_{\xi}^{\rho}: K_{\xi}^{\perp} \rightarrow K_{\xi}^{\perp}$ defined by

$$
\begin{aligned}
& T_{\xi}^{\rho}(\phi)=\left[\left(L_{\xi}^{\rho}\right)^{-1} \circ \Pi_{\xi}^{\perp} \circ i^{*}\right] M_{\xi}^{\rho}(\phi), \\
& M_{\xi}^{\rho}(\phi)=\rho^{2} V(x) \mathrm{e}^{\sum_{i=1}^{k} P U_{i}}\left[\mathrm{e}^{\phi}-1-\phi\right]+\left[\rho^{2} V(x) \mathrm{e}^{\sum_{i=1}^{k} P U_{i}}-\rho^{2} \sum_{i=1}^{k} \mathrm{e}^{U_{i}}\right] .
\end{aligned}
$$

We prove that, for $\rho$ small enough and $R$ large enough (but independent of $\rho$ ), $T_{\xi}^{\rho}$ defines a contraction map from $\left\{\|\phi\| \leqslant R \rho^{(2-p) / p}|\log \rho|\right\}$ into itself. 
Let us fix some $r>1$ such that $r(3 p-2)<2$. By Lemma 2.2 and Proposition 3.1 we have that $\left\|T_{\xi}^{\rho}(\phi)\right\| \leqslant$ $C|\log \rho|\left\|M_{\xi}^{\rho}(\phi)\right\|_{\mathrm{L}}^{p}$. Consequently, by Lemmata B.1, B.2 we deduce,

$$
\begin{aligned}
\left\|T_{\xi}^{\rho}(\phi)\right\| & \leqslant C|\log \rho|\left[\left\|\rho^{2} V(x) \mathrm{e}^{\sum_{i=1}^{k} P U_{i}}\left(\mathrm{e}^{\phi}-1-\phi\right)\right\|_{\mathrm{L}}^{p}+\left\|\rho^{2} V(x) \mathrm{e}^{\sum_{i=1}^{k} P U_{i}}-\rho^{2} \sum_{i=1}^{k} \mathrm{e}^{U_{i}}\right\|_{\mathrm{L}}^{p}\right] \\
& \leqslant C^{\prime}\|\phi\|^{2} \mathrm{e}^{c_{2}\|\phi\|^{2}} \rho^{2(1-p r) /(p r)}|\log \rho|+C^{\prime} \rho^{(2-p) / p}|\log \rho| .
\end{aligned}
$$

Hence, for $R$ suitably large we get that

$$
\|\phi\| \leqslant R \rho^{(2-p) / p}|\log \rho| \Rightarrow\left\|T_{\xi}^{\rho}(\phi)\right\| \leqslant R \rho^{(2-p) / p}|\log \rho|
$$

for $\rho>0$ sufficiently small. Similarly, by Lemma B. 2 we deduce

$$
\begin{aligned}
\left\|T_{\xi}^{\rho}\left(\phi_{1}\right)-T_{\xi}^{\rho}\left(\phi_{2}\right)\right\| & \leqslant C|\log \rho|\left\|\rho^{2} V(x) \mathrm{e}^{\sum_{i=1}^{k} P U_{i}}\left[\mathrm{e}^{\phi_{1}}-\mathrm{e}^{\phi_{2}}-\left(\phi_{1}-\phi_{2}\right)\right]\right\|_{\mathrm{L}}^{p} \\
& \leqslant C^{\prime} \rho^{(2+(2-3 p) r) /(p r)}(\log \rho)^{2}\left\|\phi_{1}-\phi_{2}\right\| \leqslant L\left\|\phi_{1}-\phi_{2}\right\|
\end{aligned}
$$

for some $L<1$, provided $\rho>0$ is small enough. By (4.4) and (4.5) we get that $T_{\xi}^{\rho}$ is a contraction map from $\left\{\|\phi\| \leqslant R \rho^{(2-p) / p}|\log \rho|\right\}$ into itself for every $\rho \in\left(0, \rho_{0}\right)$, provided $\rho_{0}>0$ is sufficiently small.

Let us introduce the functional $E_{\rho}: \mathrm{H}_{0}^{1}(\Omega) \rightarrow \mathbb{R}$ defined by

$$
E_{\rho}(u)=\frac{1}{2} \int_{\Omega}|\nabla u|^{2}-\rho^{2} \int_{\Omega} V(x) \mathrm{e}^{u} .
$$

We have the following expansion:

Proposition 4.2. Let $\widetilde{E}_{\rho}(\xi):=E_{\rho}\left(\sum_{i=1}^{k} P U_{\tau_{i}(\xi), \xi_{i}}+\phi_{\xi}^{\rho}\right)$. It holds

$$
\widetilde{E}_{\rho}(\xi)=(-16 \pi \log \rho+24 \pi \log 2-8 \pi(k+1))-32 \pi^{2} \mathcal{F}(\xi)+o(1)
$$

as $\rho \rightarrow 0, C^{1}$-uniformly in compact sets of $\left(\Omega^{\prime}\right)^{k} \backslash \Delta$ (see (1.3)).

Proof. Set $P U_{i}:=P U_{\tau_{i}(\xi), \xi_{i}}$ and $\phi:=\phi_{\xi}^{\rho}$. It holds

$$
\begin{aligned}
\widetilde{E}_{\rho}(\xi)= & \frac{1}{2} \int_{\Omega}\left|\nabla\left(\sum P U_{i}+\phi\right)\right|^{2}-\rho^{2} \int_{\Omega} V(x) \mathrm{e}^{\sum_{i=1}^{k} P U_{i}+\phi} \\
= & \frac{1}{2}\left(\sum_{i} \int_{\Omega}\left|\nabla P U_{i}\right|^{2}+\sum_{i \neq j} \int_{\Omega} \nabla P U_{i} \nabla P U_{j}\right)-\rho^{2} \int_{\Omega} V(x) \mathrm{e}^{\sum_{i=1}^{k} P U_{i}} \\
& +\frac{1}{2}\left(\int_{\Omega}|\nabla \phi|^{2}+\sum_{i} \int_{\Omega} \nabla \phi \nabla P U_{i}\right)-\rho^{2} \int_{\Omega} V(x)\left(\mathrm{e}^{\sum_{i=1}^{k} P U_{i}+\phi}-\mathrm{e}^{\sum_{i=1}^{k} P U_{i}}\right) .
\end{aligned}
$$

By the inequality $\left|\mathrm{e}^{s}-1\right| \leqslant \mathrm{e}^{|s|}|s|$ for any $s \in \mathbb{R}$ and arguing as in Lemma B.2 we obtain

$$
\left|\rho^{2} \int_{\Omega} V(x)\left(\mathrm{e}^{\sum_{i=1}^{k} P U_{i}+\phi}-\mathrm{e}^{\sum_{i=1}^{k} P U_{i}}\right)\right|=O\left(\rho^{2}\left(\int_{\Omega} \mathrm{e}^{r \sum_{i=1}^{k} P U_{i}}\right)^{1 / r}\left(\int_{\Omega} \mathrm{e}^{s|\phi|}\right)^{1 / s}\|\phi\|_{\mathrm{L}^{t}}\right)=o(1)
$$

for $r>1$ sufficiently close to 1 and $\frac{1}{r}+\frac{1}{s}+\frac{1}{t}=1$ (using (4.3)). By Lemmata C.1, C.2 and (4.8), (4.9) we deduce that expansion (4.7) holds $C^{0}$-uniformly in compact sets of $\left(\Omega^{\prime}\right)^{k} \backslash \Delta$. 
To conclude, let us make some remarks:

- as a consequence of (4.2) we have that,

$$
\sum_{i=1}^{k} P U_{i}+\phi-i^{*}\left(\rho^{2} V(x) \mathrm{e}^{\sum_{i=1}^{k} P U_{i}+\phi}\right)=\sum_{i=1}^{k} \sum_{j=1}^{2} c_{i j}^{\rho} P \psi_{i}^{j},
$$

for some coefficients $c_{i j}^{\rho}$. Inserting (4.10) into (C.15), by Lemma A.4 we deduce that

$$
\sum_{i=1}^{k} \sum_{j=1}^{2}\left|c_{i j}^{\rho}\right|=O\left(\rho^{2}\right)
$$

- the map $\xi \rightarrow \phi=\phi_{\xi}^{\rho}$ is a $C^{1}$-function as it follows by the IFT applied to the equation

$$
F(\xi, u):=\Pi_{\xi}^{\perp}\left[\sum_{i=1}^{k} P U_{i}+\Pi_{\xi}^{\perp} u-i^{*}\left(\rho^{2} V(x) \mathrm{e}^{\sum_{i=1}^{k} P U_{i}+\Pi_{\xi}^{\perp} u}\right)\right]+\Pi_{\xi} u=0 .
$$

Indeed, $F(\xi, \phi)=0$ and the linearized operator:

$$
\frac{\partial F}{\partial u}(\xi, \phi)=\Pi_{\xi}^{\perp}\left[\mathrm{Id}-i^{*}\left(\rho^{2} V(x) \mathrm{e}^{\sum_{i=1}^{k} P U_{i}+\phi} \Pi_{\xi}^{\perp}\right)\right]+\Pi_{\xi}
$$

is invertible for $\rho$ small. In fact, by (4.3) and arguing as in Lemma B.2, it is easy to derive the estimate,

$$
\left\|\Pi_{\xi}^{\perp} i^{*}\left(\rho^{2} V(x) \mathrm{e}^{\sum_{i=1}^{k} P U_{i}}\left(\mathrm{e}^{\phi}-1\right) \Pi_{\xi}^{\perp}\right)\right\|\left\|\left(L_{\xi}^{\rho}\right)^{-1}\right\|<1,
$$

for $\rho$ small. Hence, in view of Proposition 3.1 the invertibility of the operator in (4.12) easily follows.

To complete the $C^{1}$-expansion, for any $j=1, \ldots, k$ and $h=1,2$ we write

$$
\begin{aligned}
& \partial_{\left(\xi_{j}\right)_{h}} E_{\rho}\left(\sum_{i=1}^{k} P U_{i}+\phi\right) \\
& =\left(\sum_{i=1}^{k} P U_{i}+\phi-i^{*}\left(\rho^{2} V(x) \mathrm{e}^{\sum_{i=1}^{k} P U_{i}+\phi}\right), P \psi_{j}^{h}+\sum_{i=1}^{k} \partial_{\left(\xi_{j}\right)_{h}} \tau_{i}(\xi) P \psi_{i}^{0}+\partial_{\left(\xi_{j}\right)_{h}} \phi\right)_{\mathrm{H}_{0}^{1}(\Omega)} \\
& =-32 \pi^{2} \frac{\partial \mathcal{F}}{\partial\left(\xi_{j}\right)_{h}}\left(\xi_{1}, \ldots, \xi_{k}\right)+\sum_{s=1}^{k} \sum_{m=1}^{2} c_{s m}^{\rho}\left(P \psi_{s}^{m}, \sum_{i=1}^{k} \partial_{\left(\xi_{j}\right)_{h}} \tau_{i}(\xi) P \psi_{i}^{0}+\partial_{\left(\xi_{j}\right)_{h}} \phi\right)_{\mathrm{H}_{0}^{1}(\Omega)}+o(1)
\end{aligned}
$$

in view of (C.15) and (4.10). Lemma A.4 implies that

$$
\left(P \psi_{s}^{m}, P \psi_{i}^{0}\right)_{\mathrm{H}_{0}^{1}(\Omega)}=O\left(\frac{1}{\rho}\right)
$$

and the orthogonality condition $\left(P \psi_{s}^{m}, \phi\right)_{\mathrm{H}_{0}^{1}(\Omega)}=0$ implies that,

$$
\left(P \psi_{s}^{m}, \partial_{\left(\xi_{j}\right)_{h}} \phi\right)_{\mathrm{H}_{0}^{1}(\Omega)}=O\left(\|\phi\|\left\|\partial_{\left(\xi_{j}\right)_{h}} P \psi_{s}^{m}\right\|\right)=O\left(\frac{\|\phi\|}{\rho^{2}}\right)
$$

since, by a straightforward computation, we see that,

$$
\left\|\partial_{\left(\xi_{j}\right)_{h}} P \psi_{s}^{m}\right\| \leqslant\left\|\partial_{\left(\xi_{j}\right)_{h}} \psi_{s}^{m}\right\|=O\left(\frac{1}{\rho^{2}}\right)
$$

Hence, by (4.3) we get 


$$
\begin{aligned}
& \left(\sum_{i=1}^{k} P U_{i}+\phi-i^{*}\left(\rho^{2} V(x) \mathrm{e}^{\sum_{i=1}^{k} P U_{i}+\phi}\right), \sum_{i=1}^{k} \partial_{\left(\xi_{j}\right)_{h}} \tau_{i}(\xi) P \psi_{i}^{0}+\partial_{\left(\xi_{j}\right)_{h}} \phi\right)_{\mathrm{H}_{0}^{1}(\Omega)} \\
& \quad=o\left(\frac{1}{\rho^{2}} \sum_{s=1}^{k} \sum_{m=1}^{2}\left|c_{s m}^{\rho}\right|\right) .
\end{aligned}
$$

Finally, recalling (4.11), by (4.13), (4.14) we deduce

$$
\partial_{\left(\xi_{j}\right)_{h}} E_{\rho}\left(\sum_{i=1}^{k} P U_{i}+\phi\right)=-32 \pi^{2} \frac{\partial \mathcal{F}}{\partial\left(\xi_{j}\right)_{h}}\left(\xi_{1}, \ldots, \xi_{k}\right)+o(1)
$$

as $\rho \rightarrow 0$, and the proof is complete.

The finite dimensional reduction is meaningful in view of the property:

Proposition 4.3. The function $\sum_{i=1}^{k} P U_{\tau_{i}(\xi), \xi_{i}}+\phi_{\xi}^{\rho}$ is a solution of (2.1) if and only if $\xi$ is a critical point of the reduced map $\xi \rightarrow E_{\rho}\left(\sum_{i=1}^{k} P U_{\tau_{i}(\xi), \xi_{i}}+\phi_{\xi}^{\rho}\right)$.

Proof. Assume that $\xi$ is a critical point of the reduced map. Then, $\xi$ satisfies the relations

$$
\left(\sum_{i=1}^{k} P U_{i}+\phi-i^{*}\left(\rho^{2} V(x) \mathrm{e}^{\sum_{i=1}^{k} P U_{i}+\phi}\right), P \psi_{j}^{h}+\sum_{i=1}^{k} \partial_{\left(\xi_{j}\right)_{h}} \tau_{i}(\xi) P \psi_{i}^{0}+\partial_{\left(\xi_{j}\right)_{h}} \phi\right)_{\mathrm{H}_{0}^{1}(\Omega)}=0
$$

for any $j=1, \ldots, k$ and $h=1,2$. By (4.10) and (4.14) we get that,

$$
\sum_{s=1}^{k} \sum_{m=1}^{2} c_{s m}^{\rho}\left(P \psi_{s}^{m}, P \psi_{j}^{h}\right)_{\mathrm{H}_{0}^{1}(\Omega)}+o\left(\frac{1}{\rho^{2}} \sum_{s=1}^{k} \sum_{m=1}^{2}\left|c_{s m}^{\rho}\right|\right)=0 .
$$

By Lemma A.4, we conclude that $c_{s m}^{\rho}=0$ for any $s=1, \ldots, k$ and $m=1,2$, and then, by (4.10) we get

$$
\sum_{i=1}^{k} P U_{i}+\phi-i^{*}\left(\rho^{2} V(x) \mathrm{e}^{\sum_{i=1}^{k} P U_{i}+\phi}\right)=0 .
$$

The proposition now follows since the converse property is always true.

\section{Proofs of Theorem 1.1, Theorem 1.2 and some examples}

Let us recall the following notion of stability of critical points introduced in [26]:

Definition 5.1. Let $F: D \rightarrow \mathbb{R}$ be a $C^{1}$-function and let $K$ be a bounded set of critical points of $F$. We say that $K$ is a $C^{1}$-stable critical set of $F$ if for any $\mu>0$ there exists $\delta>0$ such that, if $G: D \rightarrow \mathbb{R}$ is a $C^{1}$-function with

$$
\max _{\operatorname{dist}(x, K) \leqslant \mu}(|G(x)-F(x)|+|\nabla G(x)-\nabla F(x)|) \leqslant \delta,
$$

then $G$ has at least one critical point $x$ with $\operatorname{dist}(x, K) \leqslant \mu$.

Remark 5.2. It is easy to see that a bounded set of critical point of $F$ is stable if one of the following condition is satisfied: 
(i) $K$ is a strict local maximum set of $F$, i.e. $F(x)=F(y)$ for any $x, y \in K$ and for some open neighborhood $U$ of $K$ it holds $F(x)>F(y)$ for any $x \in K$ and $y \in U \backslash K$;

(ii) $K$ is a strict local minimum set of $F$;

(iii) the Brouwer degree $\operatorname{deg}\left(\nabla F, U_{\mu}, 0\right) \neq 0$ for any $\mu>0$ small, where $U_{\mu}=\left\{x \in \mathbb{R}^{N}\right.$ : $\left.\operatorname{dist}(x, K) \leqslant \mu\right\}$.

Let us give the proof of Theorem 1.1:

Proof of Theorem 1.1. By Propositions 4.2 and 4.3, we can find a point $\xi_{\rho}=\left(\xi_{1, \rho}, \ldots, \xi_{k, \rho}\right)$ such that $u_{\rho}=$ $\sum_{i=1}^{k} P U_{\tau_{i}\left(\xi_{\rho}\right), \xi_{i, \rho}}+\phi_{\xi_{\rho}}^{\rho}$ is a solution of (2.1). Moreover, $u_{\rho}$ satisfies the "concentration" property:

$$
\rho^{2} \int_{\Omega} V(x) \mathrm{e}^{u_{\rho}} \Psi(x) \rightarrow 8 \pi \sum_{i=1}^{k} \Psi\left(q_{i}\right) \quad \text { as } \rho \rightarrow 0, \forall \Psi \in C(\bar{\Omega}) .
$$

In fact, by the inequality $\left|\mathrm{e}^{s}-1\right| \leqslant \mathrm{e}^{|s|}|s|$ for any $s \in \mathbb{R}$, we can proceed as in Proposition 4.2 to obtain

$$
\rho^{2} \int_{\Omega} V(x) \mathrm{e}^{u_{\rho}} \Psi(x)=\rho^{2} \int_{\Omega} V(x) \mathrm{e}^{\sum_{i=1}^{k} P U_{i}} \Psi(x)+o(1)=8 \pi \sum_{i=1}^{k} \Psi\left(q_{i}\right)+o(1) .
$$

Along a sequence $\rho \rightarrow 0$, we can assume that dist $\left(\xi_{\rho}, K\right) \rightarrow 0$ and the proof is completed.

Let us stress that Theorem 1.1 allows to handle the cases where the maximum of the Robin's function is not isolated (for example, in an annulus).

We are now in position to give the proof of Theorem 1.2:

Proof of Theorem 1.2. Let $k=1$. It is easy to see that $\mathcal{F}$ has a strict local maximum set in each connected component $\Omega_{i}^{\prime}$ for any $i=1, \ldots, \nu$. In fact, since $H(\xi, \xi) \rightarrow-\infty$ as $\xi \rightarrow \partial \Omega$ and $\log V(\xi) \rightarrow-\infty$ as $V(\xi) \rightarrow 0$, it follows that $\mathcal{F}(\xi) \rightarrow-\infty$ as $\xi \rightarrow \partial \Omega_{i}^{\prime}$. Therefore, the claim follows by Theorem 1.1.

In next examples, we follow some ideas introduced in [33] in order to get some contractible domains where a large number of solutions to problem (1.1) exists, provided $V(x) \equiv 1$.

Let $\Omega_{0}=\Omega_{1} \cup \ldots \cup \Omega_{h}$, where $\Omega_{1}, \ldots, \Omega_{h}$ are $h$ smooth bounded domains in $\mathbb{R}^{2}$ such that $\bar{\Omega}_{i} \cap \bar{\Omega}_{j}=\emptyset$ if $i \neq j$. Assume that

$$
\Omega_{i} \subset\left\{\left(x_{1}, x_{2}\right) \in \mathbb{R}^{2}: a_{i} \leqslant x_{1} \leqslant b_{i}\right\}, \quad \Omega_{i} \cap\left\{x_{2}=0\right\} \neq \emptyset,
$$

for some $b_{i}<a_{i+1}$ and $i=1, \ldots, h$. Let

$$
C_{\varepsilon}=\left\{\left(x_{1}, x_{2}\right) \in \mathbb{R}^{2}|| x_{2} \mid \leqslant \epsilon, x_{1} \in\left(a_{1}, b_{h}\right)\right\} .
$$

Let $\Omega_{\epsilon}$ be a smooth simply connected domain such that

$$
\Omega_{0} \subset \Omega_{\epsilon} \subset \Omega_{0} \cup C_{\epsilon} .
$$

We say that

$\Omega_{\varepsilon}$ is a $h$-dumbell.

Lemma 5.3. We have that

$$
\lim _{\epsilon \rightarrow 0} H_{\Omega_{\epsilon}}(x, x)=H_{\Omega_{0}}(x, x)
$$


$C^{1}$-uniformly on compact sets of $\Omega_{0}$ and

$$
\lim _{\epsilon \rightarrow 0} G_{\Omega_{\epsilon}}(x, y)=G_{\Omega_{0}}(x, y)
$$

$C^{1}$-uniformly on compact sets of $\Omega_{0} \times \Omega_{0} \backslash\{(x, y): x=y\}$.

Proof. We argue as in Lemma 3.2 of [33].

Theorem 5.4. For any $h \geqslant 2$ there exists an $h$-dumbell $\Omega$ for which problem (1.1) has at least $h$ different families of solutions which blow-up at a point $q_{i}$ in $\Omega, i=1, \ldots, h$, along sequences $\lambda \rightarrow 8 \pi$.

Proof. In virtue of Theorem 1.1, it is enough to find an $h$-dumbell $\Omega$, constructed as in (5.2), so that the Robin's function $H_{\Omega}(\cdot, \cdot)$ has at least $h$ disjoint stable critical sets. Since the Robin's function $H_{\Omega_{i}}(\cdot, \cdot)$ has a strict local maximum set in $\Omega_{i}$ for any $i=1, \ldots, h$, by Lemma 5.3 we deduce that if $\varepsilon$ is small enough, the Robin's function $H_{\Omega_{\varepsilon}}(\cdot, \cdot)$ has $h$ different strict local maximum sets, each contained in the corresponding $\Omega_{i}$. Since they are stable according to Definition 5.1, the claim follows.

Theorem 5.5. For any $h \geqslant 2$ there exists an $h$-dumbell $\Omega$ for which problem (1.1) has at least one family of solutions which blow-up at $h$ points $q_{1}, \ldots, q_{h}$ in $\Omega$, along a sequence $\lambda \rightarrow 8 \pi h$.

Proof. In virtue of Theorem 1.1, it is enough to find an $h$-dumbell $\Omega$, constructed as in (5.2), so that the function $\mathcal{F}_{\Omega}$, defined as

$$
\mathcal{F}_{\Omega}\left(\xi_{1}, \ldots, \xi_{h}\right)=\sum_{i=1}^{h} H_{\Omega}\left(\xi_{i}, \xi_{i}\right)+\sum_{j \neq i} G_{\Omega}\left(\xi_{j}, \xi_{i}\right),
$$

has a stable critical set according to Definition 5.1. It is easy to check that the function $\mathcal{F}_{\Omega_{0}}$ in the connected component $\Omega_{1} \times \cdots \times \Omega_{h}$ of the set $\left(\Omega_{0}\right)^{h}$ reduces to take the form:

$$
\mathcal{F}_{\Omega_{0}}\left(\xi_{1}, \ldots, \xi_{h}\right)=\sum_{i=1}^{h} H_{\Omega_{i}}\left(\xi_{i}, \xi_{i}\right),
$$

because $H_{\Omega_{0}}(x)=H_{\Omega_{i}}(x)$ if $x \in \Omega_{i}, G_{\Omega_{0}}(x, y)=G_{\Omega_{i}}(x, y)$ if $(x, y) \in \Omega_{i} \times \Omega_{i}$ and $G_{\Omega_{0}}(x, y)=0$ if $(x, y) \in$ $\Omega_{i} \times \Omega_{j}, i \neq j$. Therefore, $\mathcal{F}_{\Omega_{0}}$ has a strict local maximum set. By Lemma 5.3 we deduce that if $\varepsilon$ is small enough, the function $\mathcal{F}_{\Omega_{\epsilon}}$ also has a strict local maximum set, which is stable according to Definition 5.1, and the claim follows.

As in the previous results, we easily get:

Corollary 5.6. For any $h \geqslant 2$ there exists an h-dumbell $\Omega$ for which problem (1.1) has at least $\left(\begin{array}{l}h \\ i\end{array}\right)$ families of solutions which blow-up at $i$ points in $\Omega$ along sequences $\lambda \rightarrow 8 \pi i$, for any $i=1, \ldots, h$.

\section{Proof of Theorem 1.3 and some examples}

In this section, we will restrict to the case $V(x) \equiv 1$. Let $S, \partial Q$ and $Q$ be compact subsets of a domain $D$. We will say that $S$ links $Q$ via $\partial Q$ by homotopy in $D$ if $\partial Q \subset Q, S \cap \partial Q=\emptyset$ and $\gamma_{1}(Q) \cap S \neq \emptyset$ for any $\gamma \in \Gamma$, where

$$
\Gamma:=\left\{\gamma \in C^{0}([0,1] \times Q, D): \gamma_{0}=\mathrm{Id},\left.\gamma_{t}\right|_{\partial Q}=\operatorname{Id} \forall t \in[0,1]\right\}
$$


Now, let us recall the following notion of stability of critical values introduced in [25]. Let $F: D \rightarrow \mathbb{R}$ be a $C^{1}$-function. We say that:

Definition 6.1. $c$ is a stable critical value of $F$, if there exist compact subsets $S, \partial Q$ and $Q$ of $D$ such that $S$ links $Q$ via $\partial Q$ by homotopy in $D$,

$$
\max _{\partial Q} F<\min _{S} F
$$

and the set $\{x \in D: c-\varepsilon \leqslant F(x) \leqslant c+\varepsilon\}$ is complete for some $\varepsilon>0$, where

$$
c:=\inf _{\gamma \in \Gamma} \max _{u \in Q} F\left(\gamma_{1}(u)\right) .
$$

Proof of Theorem 1.3. By Proposition 4.3 it is enough to prove that the function $\widetilde{E}_{\rho}$ given in Proposition 4.2 has a critical point. Using the stability of $c$, the expansion (4.7) and arguing exactly as in Theorem 3.1 in [25], we can prove that if $\rho$ is small enough there exists a critical point $\xi_{\rho}$ of the function $\widetilde{E}_{\rho}$ such that $\xi_{\rho} \rightarrow \xi_{0}$ as $\rho \rightarrow 0$, with $H\left(\xi_{0}, \xi_{0}\right)=c$ and $\nabla H\left(\xi_{0}, \xi_{0}\right)=0$. The claim follows as in the proof of Theorem 1.1.

Let us prove that in a domain with "a hole" problem (1.1) has at least two families of solutions blowing-up at one point.

Theorem 6.2. Let $\Omega_{1} \subset \subset \Omega_{2}$ be smooth bounded domains and let $\Omega=\Omega_{2} \backslash \Omega_{1}$. Assume that there exists a curve $\sigma$ joining $\partial \Omega_{1}$ and $\partial \Omega_{2}$ such that

$$
\max _{\xi \in \sigma} H_{\Omega}(\xi, \xi)<\max _{\xi \in \Omega} H_{\Omega}(\xi, \xi) .
$$

Then (1.1) has at least two families of solutions which blow-up at one point in $\Omega$ as $\lambda \rightarrow 8 \pi$.

Proof. By Theorem 1.3 it is enough to prove that the Robin's function $H_{\Omega}(\cdot, \cdot)$ has at least two different stable critical values. Let $S$ be a curve contained in $\Omega$ around the hole $\Omega_{1}$. Since $H(\xi, \xi) \rightarrow-\infty$ as $\xi \rightarrow \partial \Omega$, it is clear that there exist two points $\xi_{1}$ and $\xi_{2}$ in $\sigma$ very close to $\partial \Omega_{1}$ and $\partial \Omega_{2}$, respectively, such that if $Q$ is a curve contained in $\sigma$ with endpoints $\xi_{1}$ and $\xi_{2}$ and $\partial Q:=\left\{\xi_{1}, \xi_{2}\right\}$ then

$$
\max _{\xi \in \partial Q} H(\xi, \xi)<\min _{\xi \in S} H(\xi, \xi)
$$

and moreover the curve $S$ links the segment $Q$ via $\partial Q$ by homotopy in $\Omega$. Moreover, since the Palais-Smale condition in $\Omega$ is satisfied, the Robin's function $H_{\Omega}(\cdot, \cdot)$ has a critical value $c$ such that

$$
\min _{\xi \in S} H(\xi, \xi) \leqslant c \leqslant \max _{\xi \in Q} H(\xi, \xi)<\max _{\xi \in \Omega} H(\xi, \xi)
$$

in view of (6.3). Therefore, $c$ is stable according to Definition 6.1. Finally, the claim follows, since the maximum value of the Robin's function is stable according to Definition 6.1.

Let us show that in a "2-dumbell" domain problem (1.1) has at least three families of solutions blowing-up at one point.

Theorem 6.3. Let $\Omega_{\varepsilon}$ be a 2-dumbell as in (5.2). If $\varepsilon$ is small enough then problem (1.1) has at least three families of solutions which blow-up at one point in $\Omega_{\varepsilon}$ as $\lambda \rightarrow 8 \pi$.

Proof. By Theorem 1.1 and Theorem 1.3 it is enough to prove that the function $h_{\varepsilon}(\xi):=-H_{\Omega_{\varepsilon}}(\xi, \xi)$ has at least two different stable critical points and one stable critical value. First of all, let us point out that there exist opens 
sets $B_{1} \subset \Omega_{1}, B_{2} \subset \Omega_{2}$, points $\xi_{1} \in B_{1}, \xi_{2} \in B_{2}$ and a set $S=\left\{x \in \Omega_{1} \mid \operatorname{dist}\left(x, \partial \Omega_{1}\right)=\mu\right\}$, with $\mu>0$ small enough such that

$$
h_{0}\left(\xi_{i}\right)=\min _{\Omega_{i}} h_{0}<\min _{\partial B_{i}} h_{0}, \quad i=1,2, \quad \max \left\{\min _{\partial B_{1}} h_{0}, \min _{\partial B_{2}} h_{0}\right\}<\min _{S} h_{0} .
$$

By Lemma 5.3 it follows that for $\varepsilon$ small enough it holds

$$
h_{\varepsilon}\left(\xi_{i}\right)<\min _{\partial B_{i}} h_{\varepsilon}, \quad i=1,2, \quad \max \left\{\min _{\partial B_{1}} h_{\varepsilon}, \min _{\partial B_{2}} h_{\varepsilon}\right\}<\min _{S} h_{\varepsilon} .
$$

Let $Q$ be a curve joining $\xi_{1}$ and $\xi_{2}$ in $\Omega_{\varepsilon}$ and $\partial Q=\left\{\xi_{1}, \xi_{2}\right\}$. It is clear that the curve $S$ links the curve $Q$ via $\partial Q$ by homotopy in $\Omega_{\varepsilon}$ and property (6.1) holds. Moreover, since the Palais-Smale condition in $\Omega_{\varepsilon}$ is satisfied, by (6.6) it follows that the function $h_{\varepsilon}$ has in $B_{1}$ a minimum point $\xi_{1_{\varepsilon}}$ and in $B_{2}$ a minimum point $\xi_{2_{\varepsilon}}$ (which provide two different strict local minimum sets of $h_{\varepsilon}$ ) and a stable critical value $c_{\varepsilon}$ such that $c_{\varepsilon}>\max \left\{h_{\varepsilon}\left(\xi_{1 \varepsilon}\right), h_{\varepsilon}\left(\xi_{2_{\varepsilon}}\right)\right\}$. The claim is completely established.

\section{Appendix A}

Define $\Omega_{\varepsilon}=\{\xi \in \Omega$ : $\operatorname{dist}(\xi, \partial \Omega) \geqslant 2 \varepsilon\}$ for $\varepsilon>0$. Let $P U_{\tau, \xi}$ be the projection of $U_{\tau, \xi}$ into $\mathrm{H}_{0}^{1}(\Omega)$ (see (1.5) and (1.6)).

We will estimate $P U_{\tau, \xi}$ in terms of the Robin's function and of the Green's function. We assume all along this section that $\varepsilon$ is a fixed positive number and $\tau$ varies in $(0,+\infty)$. Arguing as in [37] we can prove the following crucial estimates:

Proposition A.1. We have

$$
P U_{\tau, \xi}(x)=U_{\tau, \xi}(x)+8 \pi H(x, \xi)-\log \left(8 \tau^{2}\right)+O\left(\rho^{2}\right)
$$

in $C^{0}(\Omega)$ and in $C_{\text {loc }}^{2}(\Omega)$ as $\rho \rightarrow 0$,

$$
P U_{\tau, \xi}(x)=8 \pi G(x, \xi)+O\left(\rho^{2}\right)
$$

in $C_{\mathrm{loc}}^{0}(\bar{\Omega} \backslash\{\xi\})$ and in $C_{\mathrm{loc}}^{2}(\Omega \backslash\{\xi\})$ as $\rho \rightarrow 0$, and the convergences are uniform in $\xi \in \Omega_{\varepsilon}$ and $\tau$ bounded away from zero.

Proof. Let $\eta_{\tau, \xi}(x)=P U_{\tau, \xi}(x)-U_{\tau, \xi}(x)+\log \left(8 \tau^{2}\right)$. We have that $\eta_{\tau, \xi}$ satisfies

$$
\begin{cases}\Delta \eta_{\tau, \xi}=0 & \text { in } \Omega \\ \eta_{\tau, \xi}(x)=2 \log \left(\tau^{2} \rho^{2}+|x-\xi|^{2}\right) & \text { on } \partial \Omega\end{cases}
$$

Recalling that $\eta_{\xi}(x)=8 \pi H(x, \xi)$ verifies

$$
\begin{cases}\Delta \eta_{\xi}=0 & \text { in } \Omega \\ \eta_{\xi}(x)=2 \log |x-\xi|^{2} & \text { on } \partial \Omega\end{cases}
$$

we get

$$
\begin{cases}\Delta\left(\eta_{\tau, \xi}-\eta_{\xi}\right)=0 & \text { in } \Omega, \\ \eta_{\tau, \xi}(x)-\eta_{\xi}(x)=-2 \log \frac{|x-\xi|^{2}}{\tau^{2} \rho^{2}+|x-\xi|^{2}} & \text { on } \partial \Omega .\end{cases}
$$

Since $|x-\xi| \geqslant 2 \varepsilon$ for $x \in \partial \Omega$, by the maximum principle we get

$$
\max _{\bar{\Omega}}\left|\eta_{\tau, \xi}-\eta_{\xi}\right|=\max _{\partial \Omega}\left|\eta_{\tau, \xi}-\eta_{\xi}\right|=O\left(\rho^{2}\right)
$$


uniformly for $\xi \in \Omega_{\varepsilon}$ and $\tau$ bounded away from zero. By the representation formula for harmonic function we derive the convergence in $C_{\text {loc }}^{2}(\Omega)$. By (A.1) we deduce (A.2).

We point out the following useful estimate:

Lemma A.2. If $p \geqslant 1$, then $\left\|\rho^{2} \mathrm{e}^{U_{\tau, \xi}}\right\|_{\mathrm{L}^{p}}=O\left(\rho^{2(1-q) / p}\right)$ uniformly in $\xi \in \Omega_{\varepsilon}$ and $\tau$ bounded away from zero.

Let $P \psi_{\tau, \xi}^{j}$ be the projection into $\mathrm{H}_{0}^{1}(\Omega)$ of $\psi_{\tau, \xi}^{j}$ for $j=0,1,2$, (see (2.3)-(2.5)). Arguing as in the proof of Proposition A.1, we can prove the following crucial estimates:

Proposition A.3. If $j=0$ we have

$$
P \psi_{\tau, \xi}^{0}(x)=\psi_{\tau, \xi}^{0}(x)-\frac{2}{\tau}+O\left(\rho^{2}\right)=-4 \frac{\tau \rho^{2}}{\tau^{2} \rho^{2}+|x-\xi|^{2}}+O\left(\rho^{2}\right)
$$

in $C^{0}(\Omega)$ and in $C_{\mathrm{loc}}^{2}(\Omega)$ as $\rho \rightarrow 0$,

$$
P \psi_{\tau, \xi}^{0}(x)=O\left(\rho^{2}\right)
$$

in $C_{\mathrm{loc}}^{0}(\bar{\Omega} \backslash\{\xi\})$ and in $C_{\mathrm{loc}}^{2}(\Omega \backslash\{\xi\})$ as $\rho \rightarrow 0$, and the convergences are uniform in $\xi \in \Omega_{\varepsilon}$ and $\tau$ bounded away from zero. If $j=1,2$ we have

$$
P \psi_{\tau, \xi}^{j}(x)=\psi_{\tau, \xi}^{j}(x)+8 \pi \frac{\partial H}{\partial \xi_{j}}(x, \xi)+O\left(\rho^{2}\right)
$$

in $C^{0}(\Omega)$ and in $C_{\mathrm{loc}}^{2}(\Omega)$ as $\rho \rightarrow 0$,

$$
P \psi_{\tau, \xi}^{j}(x)=8 \pi \frac{\partial G}{\partial \xi_{j}}(x, \xi)+O\left(\rho^{2}\right)
$$

in $C_{\mathrm{loc}}^{0}(\bar{\Omega} \backslash\{\xi\})$ and in $C_{\mathrm{loc}}^{2}(\Omega \backslash\{\xi\})$ as $\rho \rightarrow 0$, and the convergences are uniform in $\xi \in \Omega_{\varepsilon}$ and $\tau$ bounded away from zero.

Finally, we have the following "orthogonality" relations:

Lemma A.4. Let $j, l=1,2$. We have that

$$
\begin{aligned}
& \left\|P \psi_{\tau, \xi}^{0}\right\|^{2}=\frac{D_{0}}{\tau^{2}}\left[1+O\left(\rho^{2}\right)\right], \\
& \left(P \psi_{\tau, \xi}^{j}, P \psi_{\tau, \xi}^{l}\right)_{\mathrm{H}_{0}^{1}(\Omega)}=\frac{D}{\tau^{2} \rho^{2}}\left[\delta_{j l}+O\left(\rho^{2}\right)\right], \\
& \left(P \psi_{\tau_{1}, \xi}^{j}, P \psi_{\tau_{2}, \xi_{2}}^{l}\right)_{\mathrm{H}_{0}^{1}(\Omega)}=O(1)
\end{aligned}
$$

as $\rho \rightarrow 0$, uniformly in $\xi, \xi_{1}, \xi_{2} \in \Omega_{\varepsilon}$ with $\left|\xi_{1}-\xi_{2}\right| \geqslant 2 \varepsilon$ and $\tau, \tau_{1}, \tau_{2}$ bounded away from zero. Here, $D_{0}=$ $64 \int_{\mathbb{R}^{2}}\left(1-|y|^{2}\right) /\left(1+|y|^{2}\right)^{4}, D=64 \int_{\mathbb{R}^{2}}|y|^{2} /\left(1+|y|^{2}\right)^{4}$ are positive constants and $\delta_{j l}$ denote the Kronecker's symbols.

Proof. Note that for $i, k=0,1,2$ 


$$
\begin{aligned}
\left(P \psi_{\tau, \xi}^{i}, P \psi_{\tau, \xi}^{k}\right)_{\mathrm{H}_{0}^{1}(\Omega)} & =\rho^{2} \int_{\Omega} \mathrm{e}^{U_{\tau, \xi}} \psi_{\tau, \xi}^{i} P \psi_{\tau, \xi}^{k} \\
& =\rho^{2} \int_{B(\xi, \varepsilon)} \mathrm{e}^{U_{\tau, \xi}} \psi_{\tau, \xi}^{i} P \psi_{\tau, \xi}^{k}+\rho^{2} \int_{\Omega \backslash B(\xi, \varepsilon)} \mathrm{e}^{U_{\tau, \xi}} \psi_{\tau, \xi}^{i} P \psi_{\tau, \xi}^{k} .
\end{aligned}
$$

We deduce by (A.3) that

$$
\begin{aligned}
\rho^{2} \int_{B(\xi, \varepsilon)} \mathrm{e}^{U_{\tau, \xi}} \psi_{\tau, \xi}^{0} P \psi_{\tau, \xi}^{0} & =16 \tau \rho^{2} \int_{B(\xi, \varepsilon)} \frac{|x-\xi|^{2}-\tau^{2} \rho^{2}}{\left(\tau^{2} \rho^{2}+|x-\xi|^{2}\right)^{3}}\left(-\frac{4 \tau \rho^{2}}{\tau^{2} \rho^{2}+|x-\xi|^{2}}+O\left(\rho^{2}\right)\right) \\
& =\frac{64}{\tau^{2}} \int_{B(0, \varepsilon / \tau \rho)} \frac{1-|y|^{2}}{\left(1+|y|^{2}\right)^{4}}+O\left(\rho^{2}\right)=\frac{D_{0}}{\tau^{2}}+O\left(\rho^{2}\right),
\end{aligned}
$$

and by (A.5) that

$$
\begin{aligned}
& \rho^{2} \int_{B(\xi, \varepsilon)} \mathrm{e}^{U_{\tau, \xi}} \psi_{\tau, \xi}^{j} P \psi_{\tau, \xi}^{l} \\
& =32 \tau^{2} \rho^{2} \int_{B(\xi, \varepsilon)} \frac{(x-\xi)_{j}}{\left(\tau^{2} \rho^{2}+|x-\xi|^{2}\right)^{3}}\left(4 \frac{(x-\xi)_{l}}{\tau^{2} \rho^{2}+|x-\xi|^{2}}+8 \pi \frac{\partial H}{\partial \xi_{l}}(\xi, \xi)+O\left(|x-\xi|+\rho^{2}\right)\right) \\
& =\frac{128}{\tau^{2} \rho^{2}} \int_{B(0, \varepsilon / \tau \rho)} \frac{y_{j} y_{l}}{\left(1+|y|^{2}\right)^{4}}+O(1)=\frac{D}{\tau^{2} \rho^{2}} \delta_{j l}+O(1) .
\end{aligned}
$$

On the other hand, by (A.4) and (A.6) we get that for $i=1, \ldots, k$

$$
\rho^{2} \int_{\Omega \backslash B(\xi, \varepsilon)} \mathrm{e}^{U_{\tau, \xi}} \psi_{\tau, \xi}^{i} P \psi_{\tau, \xi}^{i}=O\left(\rho^{2}\right)
$$

The first two estimates follow inserting (A.8)-(A.10) into (A.7). As far as the third estimate, we can write

$$
\left(P \psi_{\tau_{1}, \xi_{1}}^{j}, P \psi_{\tau_{2}, \xi_{2}}^{l}\right)_{\mathrm{H}_{0}^{1}(\Omega)}=\rho^{2} \int_{B\left(\xi_{1}, \varepsilon\right)} \mathrm{e}^{U_{\tau_{1}, \xi_{1}}} \psi_{\tau_{1}, \xi_{1}}^{j} P \psi_{\tau_{2}, \xi_{2}}^{l}+\rho^{2} \int_{\Omega \backslash B\left(\xi_{1}, \varepsilon\right)} \mathrm{e}^{U_{\tau_{1}, \xi_{1}}} \psi_{\tau_{1}, \xi_{1}}^{j} P \psi_{\tau_{2}, \xi_{2}}^{l},
$$

and by (A.6) we can estimate

$$
\begin{aligned}
& \rho^{2} \int_{B\left(\xi_{1}, \varepsilon\right)} \mathrm{e}^{U_{\tau_{1}, \xi_{1}}} \psi_{\tau_{1}, \xi_{1}}^{j} P \psi_{\tau_{2}, \xi_{2}}^{l} \\
& =8 \pi \rho^{2} \int_{B\left(\xi_{1}, \varepsilon\right)} \mathrm{e}^{U_{\tau_{1}, \xi_{1}}} \psi_{\tau_{1}, \xi_{1}}^{j}\left(\frac{\partial G}{\partial\left(\xi_{2}\right)_{l}}\left(x, \xi_{2}\right)-\frac{\partial G}{\partial\left(\xi_{2}\right)_{l}}\left(\xi_{1}, \xi_{2}\right)\right)+O(\rho)=O(1),
\end{aligned}
$$

and

$$
\rho^{2} \int_{\Omega \backslash B\left(\xi_{1}, \varepsilon\right)} \mathrm{e}^{U_{\tau_{1}, \xi_{1}}} \psi_{\tau_{1}, \xi_{1}}^{j} P \psi_{\tau_{2}, \xi_{2}}^{l}=O\left(\rho^{2}\left\|P \psi_{\tau_{2}, \xi_{2}}^{l}\right\|\right)=O(\rho) .
$$

Inserting (A.12), (A.13) into (A.11) we obtain the last estimate. 


\section{Appendix B}

In this appendix we prove some technical lemmata which allow to prove Proposition 4.1. By Proposition A.1 we deduce the following useful estimate which will give in particular an estimate of the remainder term $\phi_{\xi}^{\rho}$ in the finite dimensional reduction (see (4.3)).

Lemma B.1. Let $\xi=\left(\xi_{1}, \ldots, \xi_{k}\right) \in\left(\Omega^{\prime}\right)^{k} \backslash \Delta$ and $\tau_{i}(\xi)$ be defined as in (2.6). For any $p \geqslant 1$ there exists a positive constant $c=c(p)$, uniform in compact sets of $\left(\Omega^{\prime}\right)^{k} \backslash \Delta$, such that for any $\rho>0$ we have:

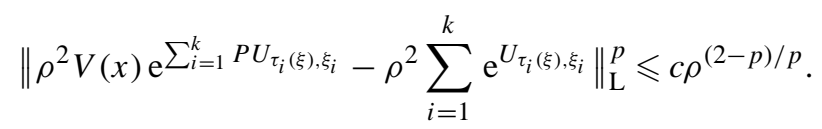

Proof. Set $U_{i}=U_{\tau_{i}(\xi), \xi_{i}}, i=1, \ldots, k$, and assume that $\xi \in \mathcal{O}_{\varepsilon}$ for some $\varepsilon>0$. We write,

$$
\begin{aligned}
& \int_{\Omega}\left|\rho^{2} V(x) \mathrm{e}^{\sum_{i=1}^{k} P U_{i}}-\rho^{2} \sum_{i=1}^{k} \mathrm{e}^{U_{i}}\right|^{p} \\
& \quad=\sum_{j=1}^{k} \int_{B\left(\xi_{j}, \varepsilon\right)}\left|\rho^{2} V(x) \mathrm{e}^{\sum_{i=1}^{k} P U_{i}}-\rho^{2} \sum_{i=1}^{k} \mathrm{e}^{U_{i}}\right|^{p}+\int_{\Omega \backslash \bigcup_{j=1}^{k} B\left(\xi_{j}, \varepsilon\right)}\left|\rho^{2} V(x) \mathrm{e}^{\sum_{i=1}^{k} P U_{i}}-\rho^{2} \sum_{i=1}^{k} \mathrm{e}^{U_{i}}\right|^{p} .
\end{aligned}
$$

By (A.2) we deduce that,

$$
\int_{\Omega \backslash \bigcup_{j=1}^{k} B\left(\xi_{j}, \varepsilon\right)}\left|\rho^{2} V(x) \mathrm{e}^{\sum_{i=1}^{k} P U_{i}}-\rho^{2} \sum_{i=1}^{k} \mathrm{e}^{U_{i}}\right|^{p}=O\left(\rho^{2 p}\right) .
$$

Moreover, for any $j=1, \ldots, k$ we have that

$$
\int_{B\left(\xi_{j}, \varepsilon\right)}\left|\rho^{2} V(x) \mathrm{e}^{\sum_{i=1}^{k} P U_{i}}-\rho^{2} \sum_{i=1}^{k} \mathrm{e}^{U_{i}}\right|^{p}=O\left(\int_{B\left(\xi_{j}, \varepsilon\right)}\left|\rho^{2} V(x) \mathrm{e}^{\sum_{i=1}^{k} P U_{i}}-\rho^{2} \mathrm{e}^{U_{j}}\right|^{p}+\rho^{2 p}\right) .
$$

By (A.1), (A.2) for $x \in B\left(\xi_{j}, \varepsilon\right), j=1, \ldots, k$, we get

$$
\sum_{i=1}^{k} P U_{i}-U_{j}=8 \pi\left(H\left(x, \xi_{j}\right)-\log \left(8 \tau_{j}^{2}\right)+\sum_{i \neq j} G\left(x, \xi_{i}\right)\right)+O\left(\rho^{2}\right)=-\log V\left(\xi_{j}\right)+O\left(\left|x-\xi_{j}\right|+\rho^{2}\right),
$$

and then, by the boundedness of $\left(\mathrm{e}^{x}-1\right) / x$ on bounded sets, finally we obtain

$$
\begin{aligned}
\int_{B\left(\xi_{j}, \varepsilon\right)}\left|\rho^{2} V(x) \mathrm{e}^{\sum_{i=1}^{k} P U_{i}}-\rho^{2} \mathrm{e}^{U_{j}}\right|^{p} & =O\left(\rho^{2 p} \int_{B\left(\xi_{j}, \varepsilon\right)} \mathrm{e}^{p U_{j}}\left(\left|x-\xi_{j}\right|+\rho^{2}\right)^{p}\right) \quad\left(\text { setting } x=\tau_{j} \rho y+\xi_{j}\right) \\
& =O\left(\rho^{2-p} \int_{B\left(0, \varepsilon / \tau_{j} \rho\right)} \frac{(|y|+\rho)^{p}}{\left(1+|y|^{2}\right)^{2 p}}\right)=O\left(\rho^{2-p}\right) .
\end{aligned}
$$

The claim follows by (B.1)-(B.4). 
Lemma B.2. Let $\varepsilon>0$. For any $p \geqslant 1$ and $r>1$ there exists positive constants $c_{1}, c_{2}$ such that for any $\rho>0$ it holds

$$
\begin{aligned}
& \| \rho^{2} V(x) \mathrm{e}^{\sum_{i=1}^{k} P U_{\tau_{i}, \xi_{i}}\left(\mathrm{e}^{\phi_{1}}-1-\phi_{1}\right)\left\|_{\mathrm{L}^{p}} \leqslant c_{1} \mathrm{e}^{c_{2}\left\|\phi_{1}\right\|^{2}} \rho^{(2-2 p r) /(p r)}\right\| \phi_{1} \|^{2},} \\
& \| \rho^{2} V(x) \mathrm{e}^{\sum_{i=1}^{k} P U_{\tau_{i}, \xi_{i}}\left(\mathrm{e}^{\phi_{1}}-\mathrm{e}^{\phi_{2}}-\left(\phi_{1}-\phi_{2}\right)\right) \|_{\mathrm{L}^{p}}} \\
& \quad \leqslant c_{1} \mathrm{e}^{c_{2}\left(\left\|\phi_{1}\right\|^{2}+\left\|\phi_{2}\right\|^{2}\right)} \rho^{(2-2 p r) /(p r)}\left\|\phi_{1}-\phi_{2}\right\|\left(\left\|\phi_{1}\right\|+\left\|\phi_{2}\right\|\right)
\end{aligned}
$$

for any $\phi_{1}, \phi_{2} \in \mathrm{H}_{0}^{1}(\Omega)$, uniformly for $\xi \in \mathcal{O}_{\varepsilon}$ and $\tau_{i}$ bounded away from zero.

Proof. Let us remark that (B.5) follows by choosing $\phi_{2}=0$ in (B.6). Let us prove (B.6). By the mean value theorem we see that, for any $\phi_{1}, \phi_{2} \in \mathbb{R}$

$$
\left|\mathrm{e}^{\phi_{1}}-\mathrm{e}^{\phi_{2}}-\left(\phi_{1}-\phi_{2}\right)\right| \leqslant \mathrm{e}^{\left|\phi_{1}\right|+\left|\phi_{2}\right|}\left|\phi_{1}-\phi_{2}\right|\left(\left|\phi_{1}\right|+\left|\phi_{2}\right|\right) .
$$

Hence, by Hölder's inequality with $\frac{1}{r}+\frac{1}{s}+\frac{1}{t}=1$ and Remark 2.4, we find that

$$
\begin{aligned}
& \left(\int_{\Omega} \mathrm{e}^{p \sum_{i=1}^{k} P U_{\tau_{i}}, \xi_{i}}\left|\mathrm{e}^{\phi_{1}}-\mathrm{e}^{\phi_{2}}-\left(\phi_{1}-\phi_{2}\right)\right|^{p}\right)^{1 / p}
\end{aligned}
$$

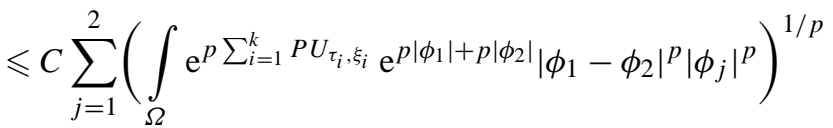

$$
\begin{aligned}
& \leqslant C \sum_{j=1}^{2}\left(\int_{\Omega} \mathrm{e}^{p r \sum_{i=1}^{k} P U_{\tau_{i}, \xi_{i}}}\right)^{1 /(p r)}\left(\int_{\Omega} \mathrm{e}^{p s\left|\phi_{1}\right|+p s\left|\phi_{2}\right|}\right)^{1 /(p s)}\left(\int_{\Omega}\left|\phi_{1}-\phi_{2}\right|^{p t}\left|\phi_{j}\right|^{p t}\right)^{1 /(p t)} \\
& \leqslant C^{\prime} \sum_{j=1}^{2}\left(\int_{\Omega} \mathrm{e}^{p r \sum_{i=1}^{k} P U_{\tau_{i}, \xi_{i}}}\right)^{1 /(p r)} \mathrm{e}^{(p s) /(8 \pi)\left(\left\|\phi_{1}\right\|^{2}+\left\|\phi_{2}\right\|^{2}\right)}\left\|\phi_{1}-\phi_{2}\right\|\left\|\phi_{j}\right\| .
\end{aligned}
$$

On the other hand, by Proposition A.1 we deduce,

$$
\left(\int_{\Omega} \mathrm{e}^{p r \sum_{i=1}^{k} P U_{\tau_{i}, \xi_{i}}}\right)^{1 /(p r)} \leqslant C^{\prime}\left(1+\sum_{i=1}^{k} \int_{B\left(\xi_{i}, \varepsilon\right)} \frac{1}{\left(\tau_{i}^{2} \rho^{2}+\left|x-\xi_{i}\right|^{2}\right)^{2 p r}}\right)^{1 /(p r)} \leqslant C \rho^{(2-4 p r) /(p r)} .
$$

Thus, (B.6) follows by means of (B.7) and (B.8).

\section{Appendix C}

This last section contains the proof of some technical lemmata. All the results we will state here are uniform in compact sets of $\left(\Omega^{\prime}\right)^{k} \backslash \Delta$. We will assume throughout the section the additional property $\xi \in \mathcal{O}_{\varepsilon}$ for some $\varepsilon>0$. For $j=1,2, i=1, \ldots, k$ set $U_{i}:=U_{\tau_{i}(\xi), \xi_{i}}$ and $\psi_{i}^{j}:=\psi_{\tau_{i}(\xi), \xi_{i}}^{j}$, where $\tau_{i}=\tau_{i}(\xi)$ is defined in (2.6).

Lemma C.1. We have:

$$
\int_{\Omega}\left|\nabla P U_{i}\right|^{2}=(-32 \pi \log \rho+48 \pi \log 2-16 \pi)-64 \pi^{2} \mathcal{F}(\xi)-64 \pi^{2} \sum_{j \neq i} G\left(\xi_{i}, \xi_{j}\right)+O(\rho)
$$

and 


$$
\int_{\Omega} \nabla P U_{i} \nabla P U_{j}=64 \pi^{2} G\left(\xi_{i}, \xi_{j}\right)+O(\rho)
$$

as $\rho \rightarrow 0$.

Proof. By Proposition A.1 we get that

$$
\begin{aligned}
\int_{\Omega}\left|\nabla P U_{i}\right|^{2}= & \rho^{2} \int_{\Omega} \mathrm{e}^{U_{i}} P U_{i} \\
= & \int_{B\left(\xi_{i}, \varepsilon\right)} \frac{8 \tau_{i}^{2}(\xi) \rho^{2}}{\left(\tau_{i}^{2}(\xi) \rho^{2}+\left|x-\xi_{i}\right|^{2}\right)^{2}}\left(-2 \log \left(\tau_{i}^{2}(\xi) \rho^{2}+\left|x-\xi_{i}\right|^{2}\right)\right. \\
& \left.+8 \pi H\left(\xi_{i}, \xi_{i}\right)+O\left(\left|x-\xi_{i}\right|+\rho^{2}\right)\right)+O\left(\rho^{2}\right) \quad\left(\operatorname{setting} x=\tau_{i}(\xi) \rho y+\xi_{i}\right) \\
= & \int_{B\left(0, \varepsilon / \tau_{i}(\xi) \rho\right)} \frac{8}{\left(1+|y|^{2}\right)^{2}}(-4 \log \rho+6 \log 2-8 \pi \mathcal{F}(\xi) \\
& \left.-8 \pi \sum_{j \neq i} G\left(\xi_{i}, \xi_{j}\right)-2 \log \left(1+|y|^{2}\right)\right)+O(\rho) \\
= & (-32 \pi \log \rho+48 \pi \log 2-16 \pi)-64 \pi^{2} \mathcal{F}(\xi)-64 \pi^{2} \sum_{j \neq i} G\left(\xi_{i}, \xi_{j}\right)+O(\rho),
\end{aligned}
$$

where we have used the fact that we have the relation:

$$
\int_{\mathbb{R}^{2}} \frac{1}{\left(1+|y|^{2}\right)^{2}} \log \left(1+|y|^{2}\right)=\int_{\mathbb{R}^{2}} \frac{1}{\left(1+|y|^{2}\right)^{2}}=\pi
$$

(by an integration by parts). By (C.3), in view of Proposition A.1 for $i \neq j$ we obtain

$$
\begin{aligned}
\int_{\Omega} \nabla P U_{i} \nabla P U_{j} & =\int_{B\left(\xi_{i}, \varepsilon\right)} \frac{8 \tau_{i}^{2}(\xi) \rho^{2}}{\left(\tau_{i}^{2}(\xi) \rho^{2}+\left|x-\xi_{i}\right|^{2}\right)^{2}}\left(8 \pi G\left(\xi_{i}, \xi_{j}\right)+O\left(\left|x-\xi_{i}\right|+\rho^{2}\right)\right)+O\left(\rho^{2}\left\|P U_{j}\right\|\right) \\
& =64 \pi^{2} G\left(\xi_{i}, \xi_{j}\right)+O(\rho) .
\end{aligned}
$$

Lemma C.2. We have:

$$
\rho^{2} \int_{\Omega} V(x) \mathrm{e}^{\sum_{i} P U_{i}}=8 \pi k+o(1)
$$

as $\rho \rightarrow 0$.

Proof. By Proposition A.1 we get

$$
\begin{aligned}
\rho^{2} \int_{\Omega} V(x) \mathrm{e}^{\sum_{h=1}^{k} P U_{h}} & =\sum_{i=1}^{k} \rho^{2} \int_{B\left(\xi_{i}, \varepsilon\right)} V(x) \mathrm{e}^{\sum_{h=1}^{k} P U_{h}}+O\left(\rho^{2}\right) \\
& =\sum_{i=1}^{k} \int_{B\left(\xi_{i}, \varepsilon\right)} \frac{\rho^{2} V\left(\xi_{i}\right)}{\left(\tau_{i}^{2} \rho^{2}+\left|x-\xi_{i}\right|^{2}\right)^{2}} \mathrm{e}^{8 \pi\left(H\left(\xi_{i}, \xi_{i}\right)+\sum_{h \neq i} G\left(\xi_{i}, \xi_{h}\right)\right)}
\end{aligned}
$$




$$
\begin{aligned}
& \times\left(1+O\left(\left|x-\xi_{i}\right|+\rho^{2}\right)\right)+O\left(\rho^{2}\right) \quad\left(\text { setting } x=\tau_{i} \rho y+\xi_{i}\right) \\
= & \sum_{i=1}^{k} \int_{B\left(0, \varepsilon / \tau_{i} \rho\right)} \frac{8}{\left(1+|y|^{2}\right)^{2}} d y+o(1)=8 \pi k+o(1) .
\end{aligned}
$$

Lemma C.3. Let $j=1,2$ and $i, h=1, \ldots, k$. We have:

$$
\rho^{2} \int_{\Omega} \mathrm{e}^{U_{i}} P \psi_{h}^{j}=64 \pi^{2} \frac{\partial H}{\partial\left(\xi_{i}\right)_{j}}\left(\xi_{i}, \xi_{i}\right) \delta_{i h}+64 \pi^{2} \frac{\partial G}{\partial\left(\xi_{h}\right)_{j}}\left(\xi_{i}, \xi_{h}\right)\left(1-\delta_{i h}\right)+O(\rho)
$$

as $\rho \rightarrow 0$ (here $\delta_{i h}$ is the Kronecker's symbol).

Proof. Since

$$
\rho^{2} \int_{\Omega} \mathrm{e}^{U_{i}} P \psi_{h}^{j}=\rho^{2} \int_{\Omega \backslash B\left(\xi_{i}, \varepsilon\right)} \mathrm{e}^{U_{i}} P \psi_{h}^{j}+\rho^{2} \int_{B\left(\xi_{i}, \varepsilon\right)} \mathrm{e}^{U_{i}} P \psi_{h}^{j}
$$

and, by Lemma A.4,

$$
\rho^{2} \int_{\Omega \backslash B\left(\xi_{i}, \varepsilon\right)} \mathrm{e}^{U_{i}} P \psi_{h}^{j}=O(\rho)
$$

we are left to estimate the second term in (C.6). By (A.5) we find,

$$
\begin{aligned}
\rho^{2} \int_{B\left(\xi_{i}, \varepsilon\right)} \mathrm{e}^{U_{i}} P \psi_{i}^{j} & =\rho^{2} \int_{B\left(\xi_{i}, \varepsilon\right)} \mathrm{e}^{U_{i}}\left(4 \frac{\left(x-\xi_{i}\right)_{j}}{\tau_{i}^{2} \rho^{2}+\left|x-\xi_{i}\right|^{2}}+8 \pi \frac{\partial H}{\partial\left(\xi_{i}\right)_{j}}\left(x, \xi_{i}\right)+O\left(\rho^{2}\right)\right) \\
& =8 \pi \frac{\partial H}{\partial\left(\xi_{i}\right)_{j}}\left(\xi_{i}, \xi_{i}\right) \rho^{2} \int_{B\left(\xi_{i}, \varepsilon\right)} \mathrm{e}^{U_{i}}+O(\rho)=64 \pi^{2} \frac{\partial H}{\partial\left(\xi_{i}\right)_{j}}\left(\xi_{i}, \xi_{i}\right)+O(\rho)
\end{aligned}
$$

since $\int_{B\left(\xi_{i}, \varepsilon\right)} \mathrm{e}^{U_{i}}\left(x-\xi_{i}\right)_{j} /\left(\tau_{i}^{2} \rho^{2}+\left|x-\xi_{i}\right|^{2}\right)=0$ by symmetry, and (A.6) provides for $i \neq h$

$$
\begin{aligned}
\rho^{2} \int_{B\left(\xi_{i}, \varepsilon\right)} \mathrm{e}^{U_{i}} P \psi_{h}^{j} & =\rho^{2} \int_{B\left(\xi_{i}, \varepsilon\right)} \mathrm{e}^{U_{i}}\left(8 \pi \frac{\partial G}{\partial\left(\xi_{h}\right)_{j}}\left(x, \xi_{h}\right)+O\left(\rho^{2}\right)\right)=8 \pi \frac{\partial G}{\partial\left(\xi_{h}\right)_{j}}\left(\xi_{i}, \xi_{h}\right) \rho^{2} \int_{B\left(\xi_{i}, \varepsilon\right)} e^{U_{i}}+O(\rho) \\
& =64 \pi^{2} \frac{\partial G}{\partial\left(\xi_{h}\right)_{j}}\left(\xi_{i}, \xi_{h}\right)+O(\rho) .
\end{aligned}
$$

Thus, (C.5) follows by (C.6)-(C.9).

Lemma C.4. Let $j=1,2$ and $i=1, \ldots, k$. We have:

$$
\begin{aligned}
& \rho^{2} \int_{\Omega} V(x) \mathrm{e}^{\sum_{h=1}^{k} P U_{h}} P \psi_{i}^{j} \\
& \quad=64 \pi^{2} \frac{\partial}{\partial\left(\xi_{i}\right)_{j}}\left(\sum_{l=1}^{k} H\left(\xi_{l}, \xi_{l}\right)+\sum_{l \neq h} G\left(\xi_{l}, \xi_{h}\right)+\frac{1}{8 \pi} \sum_{l=1}^{k} \log V\left(\xi_{l}\right)\right)+O(\rho)
\end{aligned}
$$

as $\rho \rightarrow 0$. 
Proof. Write,

$$
\begin{aligned}
\rho^{2} \int_{\Omega} V(x) \mathrm{e}^{\sum_{h=1}^{k} P U_{h}} P \psi_{i}^{j}= & \rho^{2} \int_{B\left(\xi_{i}, \varepsilon\right)} V(x) \mathrm{e}^{\sum_{h=1}^{k} P U_{h}} P \psi_{i}^{j}+\rho^{2} \sum_{l \neq i} \int_{B\left(\xi_{l}, \varepsilon\right)} V(x) \mathrm{e}^{\sum_{h=1}^{k} P U_{h}} P \psi_{i}^{j} \\
& +\rho^{2} \int_{\Omega \backslash \bigcup_{l=1}^{k} B\left(\xi_{l}, \varepsilon\right)} V(x) \mathrm{e}^{\sum_{h=1}^{k} P U_{h}} P \psi_{i}^{j},
\end{aligned}
$$

and by (A.2), Lemma A.4 deduce that,

$$
\rho^{2}\left|\int_{\Omega \backslash \bigcup_{l=1}^{k} B\left(\xi_{l}, \varepsilon\right)} V(x) \mathrm{e}^{\sum_{h=1}^{k} P U_{h}} P \psi_{i}^{j}\right| \leqslant C \rho^{2} \int_{\Omega}\left|P \psi_{i}^{j}\right|=O(\rho) .
$$

By Propositions A.1, A.3 we can also estimate the first term in (C.11) as follows:

$$
\begin{aligned}
\rho^{2} & \int_{B\left(\xi_{i}, \varepsilon\right)} V(x) \mathrm{e}^{\sum_{h=1}^{k} P U_{h}} P \psi_{i}^{j} \\
= & \rho^{2} \int_{B\left(\xi_{i}, \varepsilon\right)} \frac{V(x) \mathrm{e}^{8 \pi\left(H\left(x, \xi_{i}\right)+\sum_{h \neq i} G\left(x, \xi_{h}\right)\right)}}{\left(\tau_{i}^{2} \rho^{2}+\left|x-\xi_{i}\right|^{2}\right)^{2}}\left(4 \frac{\left(x-\xi_{i}\right)_{j}}{\tau_{i}^{2} \rho^{2}+\left|x-\xi_{i}\right|^{2}}+8 \pi \frac{\partial H}{\partial\left(\xi_{i}\right)_{j}}\left(x, \xi_{i}\right)+O(\rho)\right) \\
= & \int_{B\left(0, \varepsilon /\left(\tau_{i} \rho\right)\right)} \frac{V\left(\tau_{i} \rho y+\xi_{i}\right) \mathrm{e}^{8 \pi\left(H\left(\tau_{i} \rho y+\xi_{i}, \xi_{i}\right)+\sum_{h \neq i} G\left(\tau_{i} \rho y+\xi_{i}, \xi_{h}\right)\right)}}{\tau_{i}^{2}\left(1+|y|^{2}\right)^{2}}\left(\frac{4}{\tau_{i} \rho} \frac{y_{j}}{1+|y|^{2}}+8 \pi \frac{\partial H}{\partial\left(\xi_{i}\right)_{j}}\left(\xi_{i}, \xi_{i}\right)\right) \\
& +O(\rho) \\
= & \frac{32}{\tau_{i} \rho} \int_{B\left(0, \varepsilon /\left(\tau_{i} \rho\right)\right)}\left[1+\tau_{i} \rho \sum_{s=1}^{2} y_{s} \frac{\partial}{\partial\left(\xi_{i}\right)_{s}}\left(4 \pi H\left(\xi_{i}, \xi_{i}\right)+8 \pi \sum_{h \neq i} G\left(\xi_{i}, \xi_{h}\right)+\log V\left(\xi_{i}\right)\right)\right] \frac{y_{j}}{\left(1+|y|^{2}\right)^{3}} \\
& +32 \pi \frac{\partial}{\partial\left(\xi_{i}\right)_{j}}\left(H\left(\xi_{i}, \xi_{i}\right)\right) \int_{B\left(0, \varepsilon /\left(\tau_{i} \rho\right)\right)} \frac{1}{\left(1+|y|^{2}\right)^{2}}+O(\rho) \\
= & 64 \pi^{2} \frac{\partial}{\partial\left(\xi_{i}\right)_{j}}\left(H\left(\xi_{i}, \xi_{i}\right)+\sum_{h \neq i} G\left(\xi_{i}, \xi_{h}\right)+\frac{1}{8 \pi} \log V\left(\xi_{i}\right)\right)+O(\rho) .
\end{aligned}
$$

If $l \neq i$, by Propositions A.1, A. 3 we get

$$
\begin{aligned}
& \rho^{2} \int_{B\left(\xi_{l}, \varepsilon\right)} V(x) \mathrm{e}^{\sum_{h=1}^{k} P U_{h}} P \psi_{i}^{j}=\rho^{2} \int_{B\left(\xi_{l}, \varepsilon\right)} \frac{V(x) \mathrm{e}^{8 \pi\left(H\left(x, \xi_{l}\right)+\sum_{h \neq l} G\left(x, \xi_{h}\right)\right)}}{\left(\tau_{l}^{2} \rho^{2}+\left|x-\xi_{l}\right|^{2}\right)^{2}}\left(8 \pi \frac{\partial G}{\partial\left(\xi_{i}\right)_{j}}\left(x, \xi_{i}\right)+O\left(\rho^{2}\right)\right) \\
& =8 \pi \int_{B\left(0, \varepsilon /\left(\tau_{l} \rho\right)\right)} \frac{V\left(\tau_{l} \rho y+\xi_{l}\right) \mathrm{e}^{8 \pi\left(H\left(\tau_{l} \rho y+\xi_{l}, \xi_{l}\right)+\sum_{h \neq l} G\left(\tau_{l} \rho y+\xi_{l}, \xi_{h}\right)\right)}}{\tau_{l}^{2}\left(1+|y|^{2}\right)^{2}} \frac{\partial G}{\partial\left(\xi_{i}\right)_{j}}\left(\tau_{l} \rho y+\xi_{l}, \xi_{i}\right)+O\left(\rho^{2}\right) \\
& =64 \pi^{2} \frac{\partial G}{\partial\left(\xi_{i}\right)_{j}}\left(\xi_{l}, \xi_{i}\right)+O(\rho) .
\end{aligned}
$$

In view of (C.11), by (C.12)-(C.14) we derive (C.10). 
Lemma C.5. It holds

$$
\left(\sum_{h=1}^{k} P U_{h}+\phi-i^{*}\left(\rho^{2} V(x) \mathrm{e}^{\sum_{h=1}^{k} P U_{h}+\phi}\right), P \psi_{i}^{j}\right)_{\mathrm{H}_{0}^{1}(\Omega)}=-32 \pi^{2} \frac{\partial \mathcal{F}}{\partial\left(\xi_{i}\right)_{j}}\left(\xi_{1}, \ldots, \xi_{k}\right)+o(1),
$$

as $\rho \rightarrow 0, C^{1}$-uniformly for $\xi$ in a compact set of $\left(\Omega^{\prime}\right)^{k} \backslash \Delta$.

Proof. Let $p \in\left(1, \frac{6}{5}\right)$ be fixed. We have,

$$
\begin{aligned}
\int_{\Omega} & \nabla \\
& \left(\sum_{h=1}^{k} P U_{h}+\phi\right) \nabla P \psi_{i}^{j}-\rho^{2} \int_{\Omega} V(x) \mathrm{e}^{\sum_{h=1}^{k} P U_{h}+\phi} P \psi_{i}^{j} \\
= & \sum_{h=1}^{k} \int_{\Omega} \nabla P U_{h} \nabla P \psi_{i}^{j}-\rho^{2} \int_{\Omega} V(x) \mathrm{e}^{\sum_{h=1}^{k} P U_{h}} P \psi_{i}^{j} \\
& -\rho^{2} \int_{\Omega} V(x) \mathrm{e}^{\sum_{h=1}^{k} P U_{h}} \phi P \psi_{i}^{j}-\rho^{2} \int_{\Omega} V(x) \mathrm{e}^{\sum_{h=1}^{k} P U_{h}}\left(\mathrm{e}^{\phi}-1-\phi\right) P \psi_{i}^{j} \\
= & \rho^{2} \sum_{h=1}^{k} \int_{\Omega} \mathrm{e}^{U_{h}} P \psi_{i}^{j}-\rho^{2} \int_{\Omega} V(x) \mathrm{e}^{\sum_{h=1}^{k} P U_{h} P \psi_{i}^{j}} \\
& +\int_{\Omega} \int_{\left.\rho^{2} \sum_{h=1}^{k} \mathrm{e}^{U_{h}}-\rho^{2} V(x) \mathrm{e}^{\sum_{h=1}^{k} P U_{h}}\right) \phi P \psi_{i}^{j}+\rho^{2} \int_{\Omega} \mathrm{e}^{U_{i}} \phi\left(\psi_{i}^{j}-P \psi_{i}^{j}\right)} \\
& -\rho^{2} \sum_{h \neq i} \int_{\Omega} \mathrm{e}^{U_{h}} \phi P \psi_{i}^{j}-\rho^{2} \int_{\Omega} V(x) \mathrm{e}^{\sum_{h=1}^{k} P U_{h}}\left(\mathrm{e}^{\phi}-1-\phi\right) P \psi_{i}^{j} .
\end{aligned}
$$

In view of Lemmata C.3, C.4 we deduce that

$$
\rho^{2} \sum_{h=1}^{k} \int_{\Omega} \mathrm{e}^{U_{h}} P \psi_{i}^{j}-\rho^{2} \int_{\Omega} V(x) \mathrm{e}^{\sum_{h=1}^{k} P U_{h}} P \psi_{i}^{j}=-32 \pi^{2} \frac{\partial \mathcal{F}}{\partial\left(\xi_{i}\right)_{j}}\left(\xi_{1}, \ldots, \xi_{k}\right)+O(\rho) .
$$

Moreover, with the aid of Hölder's inequality (here $1 / q+1 / p<1$ ), we can use Lemmata A.4, B.1 to find,

$$
\begin{aligned}
\left|\int_{\Omega}\left(\rho^{2} \sum_{h=1}^{k} \mathrm{e}^{U_{h}}-\rho^{2} V(x) \mathrm{e}^{\sum_{h=1}^{k} P U_{h}}\right) \phi P \psi_{i}^{j}\right| & \leqslant c\|\phi\|_{\mathrm{L}^{q}}\left\|\rho^{2} \sum_{h=1}^{k} \mathrm{e}^{U_{h}}-\rho^{2} V(x) \mathrm{e}^{\sum_{h=1}^{k} P U_{h}}\right\|\left\|_{\mathrm{L}^{p}}\right\| P \psi_{i}^{j} \| \\
& \leqslant c\|\phi\| \rho^{2(1-p) / p} ;
\end{aligned}
$$

while, by Lemma C.7 we get,

$$
\left|\int_{\Omega} \rho^{2} \mathrm{e}^{U_{i}} \phi\left(\psi_{i}^{j}-P \psi_{i}^{j}\right)\right| \leqslant c\|\phi\|_{\mathrm{L}^{q}}\left\|\rho^{2} \mathrm{e}^{U_{i}}\left(\psi_{i}^{j}-P \psi_{i}^{j}\right)\right\|_{\mathrm{L}^{p}} \leqslant c\|\phi\| \rho^{2(1-p) / r p},
$$

and Lemma C.6 provides

$$
\left|\rho^{2} \sum_{h \neq i} \int_{\Omega} \mathrm{e}^{U_{h}} \phi P \psi_{i}^{j}\right| \leqslant c\|\phi\|_{\mathrm{L}^{q}} \sum_{h \neq i}\left\|\rho^{2} \mathrm{e}^{U_{h}} P \psi_{i}^{j}\right\|_{\mathrm{L}^{p}} \leqslant c\|\phi\| \rho^{2(1-p) / p} .
$$


Finally, Lemmata A.4, B.2 give,

$$
\begin{aligned}
& \rho^{2}\left|\int_{\Omega} V(x) \mathrm{e}^{\sum_{h=1}^{k} P U_{h}}\left(\mathrm{e}^{\phi}-1-\phi\right) P \psi_{i}^{j}\right| \\
& \quad \leqslant c\left\|\rho^{2} V(x) \mathrm{e}^{\sum_{h=1}^{k} P U_{h}}\left(\mathrm{e}^{\phi}-1-\phi\right)\right\|_{\mathrm{L}^{p}}\left\|P \psi_{i}^{j}\right\|_{\mathrm{L}^{q}} \leqslant c\|\phi\|^{2} \rho^{(2-3 p r) /(p r)},
\end{aligned}
$$

for some $r>1$. We fix $r>1$ sufficiently close to 1 so that $2+4 r-5 p r>0$. Inserting the estimate (4.3) in (C.18)-(C.21) and taking in account (C.17), by (C.16) the claim in (C.15) follows.

Lemma C.6. Let $j=1,2$ and $i, h=1, \ldots, k$. If $p \geqslant 1$ and $i \neq h$, then

$$
\left\|\rho^{2} \mathrm{e}^{U_{i}} P \psi_{h}^{j}\right\|_{\mathrm{L}^{p}}=O\left(\rho^{2(1-p) / p}\right)
$$

as $\rho \rightarrow 0$.

Proof. We have

$$
\rho^{2 p} \int_{\Omega}\left|\mathrm{e}^{U_{i}} P \psi_{h}^{j}\right|^{p}=\rho^{2 p} \int_{B\left(\xi_{i}, \varepsilon\right)}\left|\mathrm{e}^{U_{i}} P \psi_{h}^{j}\right|^{p}+\rho^{2 p} \int_{\Omega \backslash B\left(\xi_{i}, \varepsilon\right)}\left|\mathrm{e}^{U_{i}} P \psi_{h}^{j}\right|^{p} .
$$

By Lemma A.4 we get

$$
\rho^{2 p} \int_{\Omega \backslash B\left(\xi_{i}, \varepsilon\right)}\left|\mathrm{e}^{U_{i}} P \psi_{h}^{j}\right|^{p}=O\left(\rho^{p}\right) .
$$

By (A.6) and Lemma A.2 we derive

$$
\rho^{2 p} \int_{B\left(\xi_{i}, \varepsilon\right)}\left|\mathrm{e}^{U_{i}} P \psi_{h}^{j}\right|^{p}=O\left(\left\|\rho^{2} \mathrm{e}^{U_{i}}\right\|_{\mathrm{L}^{p}}^{p}\right)=O\left(\rho^{2-2 p}\right) .
$$

By (C.22)-(C.24) we obtain the desired estimate.

Lemma C.7. Let $j=1,2$ and $i=1, \ldots, k$. If $p \geqslant 1$, then

$$
\left\|\rho^{2} \mathrm{e}^{U_{i}}\left(P \psi_{i}^{j}-\psi_{i}^{j}\right)\right\|_{\mathrm{L}^{p}}=O\left(\rho^{2(1-p) / p}\right)
$$

as $\rho \rightarrow 0$.

Proof. By (A.5) we obtain that $P \psi_{i}^{j}-\psi_{i}^{j}=O(1)$, and by Lemma A.2 we get,

$$
\left\|\rho^{2} \mathrm{e}^{U_{i}}\left(P \psi_{i}^{j}-\psi_{i}^{j}\right)\right\|_{\mathrm{L}^{p}}=O\left(\left\|\rho^{2} \mathrm{e}^{U_{i}}\right\|_{\mathrm{L}^{p}}\right)=O\left(\rho^{2(1-p) / p}\right) .
$$

\section{Appendix D}

We are interested to prove a result concerning the structure of the solutions of the linearized problem "at infinity". This information will be crucial in the proof of Proposition 3.1. The corresponding result in higher dimensions is well known (cf. [1,7,37]). Here, we use some ideas of [20] (see also [1] and [18]). We recall that this result was first stated in [14] for solution in $L^{\infty}\left(\mathbb{R}^{2}\right)$. 
Lemma D.1. Let $U \in C^{2}\left(\mathbb{R}^{2}\right)$ be a solution of the following problem

$$
\left\{\begin{array}{l}
-\Delta U=\frac{8}{\left(1+|y|^{2}\right)^{2}} U \text { in } \mathbb{R}^{2} \\
\int_{\mathbb{R}^{2}}|\nabla U|^{2} \mathrm{~d} y<+\infty
\end{array}\right.
$$

Then,

$$
U(y)=a_{0} \frac{1-|y|^{2}}{1+|y|^{2}}+\sum_{i=1}^{2} a_{i} \frac{y_{i}}{1+|y|^{2}}
$$

for some real numbers $a_{i}, i=0,1,2$.

Proof. We expand $U$ in Fourier series as follows,

$$
U(y)=a_{0}(r)+\sum_{k=1}^{\infty}\left(a_{k}(r) \cos (k \theta)+b_{k}(r) \sin (k \theta)\right), \quad y=r(\cos \theta, \sin \theta) .
$$

Thus, (D.1) reduces to study, for any $k \geqslant 0$, the ordinary differential equation

$$
-\ddot{u}(r)-\frac{1}{r} \dot{u}(r)+\frac{k^{2}}{r^{2}} u(r)=\frac{8}{\left(1+r^{2}\right)^{2}} u(r) \quad \text { in }(0,+\infty)
$$

with the integral condition

$$
\int_{0}^{\infty} \dot{u}(r)^{2} r \mathrm{~d} r<+\infty
$$

Let us consider the case $k=0$. A direct computation shows that $\zeta_{0}(r)=\left(1-r^{2}\right) /\left(1+r^{2}\right)$ is a solution of (D.2) $\left.\right|_{k=0}$ which satisfies (D.3). Let us prove that, if $w$ is a second linearly independent solution of (D.2) $\left.\right|_{k=0}$, then $w$ verifies $\int_{0}^{\infty} \dot{w}(r)^{2} r \mathrm{~d} r=+\infty$. Writing $w(r)=c(r) \zeta_{0}(r)$ for $r<1$, we get that

$$
-\ddot{c} \zeta_{0}-\dot{c}\left(2 \dot{\zeta}_{0}+\frac{1}{r} \zeta_{0}\right)=0
$$

and so,

$$
\begin{aligned}
& \dot{c}(r)=\frac{C}{r \zeta_{0}^{2}(r)}=C \frac{\left(1+r^{2}\right)^{2}}{r\left(1-r^{2}\right)^{2}} \sim \frac{C}{r} \quad \text { for } r \text { small, } \\
& c(r) \sim C \log r \quad \text { for } r \text { small, }
\end{aligned}
$$

where $C \neq 0$ is a constant. Hence, $\dot{w}(r) \sim \frac{C}{r}$ for $r$ small and $\int_{0}^{\infty} \dot{w}(r)^{2} r \mathrm{~d} r=+\infty$. Now we consider the case $k=1$ in (D.2). Here, we have that $\zeta_{1}(r)=\frac{r}{1+r^{2}}$ is a solution of (D.2) $\left.\right|_{k=1}$ which satisfies (D.3). As in case $k=0$, we obtain that, for a second linearly independent solution $w(r)$ in the form $w(r)=c(r) \zeta_{1}(r)$ the function $c(r)$ must satisfy

$$
\dot{c}(r)=\frac{C}{r \zeta_{1}(r)^{2}} \sim \frac{C}{r^{3}} \quad \text { and } \quad c(r) \sim-\frac{C}{2 r^{2}} \quad \text { for } r \text { small, }
$$

where $C \neq 0$ is a constant. Hence, $\dot{w}(r) \sim C /\left(2 r^{2}\right)$ for $r$ small and $\int_{0}^{\infty} \dot{w}(r)^{2} r \mathrm{~d} r=+\infty$. Now, let us show that (D.2) $\left.\right|_{k}$ for $k \geqslant 2$ has no nontrivial solution satisfying (D.3). In fact,

$$
\zeta_{k}^{1}(r)=\frac{(k+1)+(k-1) r^{2}}{1+r^{2}} r^{k} \quad \text { and } \quad \zeta_{k}^{2}(r)=\frac{(k-1)+(k+1) r^{2}}{1+r^{2}} r^{-k}
$$


for $k \geqslant 2$ represent a set of fundamental solutions for (D.2) $\left.\right|_{k}$ and they do not satisfy the integral condition (D.3) at infinity and at the origin respectively.

Hence,

$$
\begin{aligned}
U(y) & =a_{0}(r)+a_{1}(r) \cos \theta+b_{1}(r) \sin \theta=a_{0} \zeta_{0}(r)+a_{1} \zeta_{1}(r) \cos \theta+a_{2} \zeta_{1}(r) \sin \theta \\
& =a_{0} \frac{1-|y|^{2}}{1+|y|^{2}}+\sum_{i=1}^{2} a_{i} \frac{y_{i}}{1+|y|^{2}},
\end{aligned}
$$

where $a_{0}, a_{1}, a_{2}$ are real numbers.

\section{References}

[1] A. Ambrosetti, J. Garcia Azorero, I. Peral, Perturbation of $\Delta u+u^{(N+2) /(N-2)}=0$, the scalar curvature problem in $\mathbb{R}^{N}$, and related topics, J. Funct. Anal. 165 (1999) 117-149.

[2] T. Aubin, Some Nonlinear Problems in Riemannian Geometry, Springer-Verlag, Berlin, 1998.

[3] A. Bahri, Critical Point at Infinity in Some Variational Problems, Pitman Research Notes Math., vol. 182, Longman House, Harlow, 1989.

[4] C. Bandle, Isoperimetric Inequalities and Applications, Pitman Monographs Studies Math., vol. 7, Pitman, 1980.

[5] S. Baraket, F. Pacard, Construction of singular limits for a semilinear elliptic equation in dimension 2, Calc. Var. Partial Differential Equations 6 (1998) 1-38.

[6] J. Bebernes, D. Eberly, Mathematical Problems from Combustion Theory, Springer, Berlin, 1989.

[7] G. Bianchi, H. Egnell, A note on the Sobolev inequality, J. Funct. Anal. 100 (1991) 18-24.

[8] H. Brezis, F. Merle, Uniform estimates and blow-up behavior for solutions of $-\Delta u=V(x) \mathrm{e}^{u}$ in two dimensions, Comm. Partial Differential Equations 16 (1991) 1223-1253.

[9] E. Caglioti, P.L. Lions, C. Marchioro, M. Pulvirenti, A special class of stationary flows for two-dimensional Euler equations: a statistical mechanics description, Comm. Math. Phys. 143 (1992) 501-525.

[10] E. Caglioti, P.L. Lions, C. Marchioro, M. Pulvirenti, A special class of stationary flows for two-dimensional Euler equations: a statistical mechanics description. Part II, Comm. Math. Phys. 174 (1995) 229-260.

[11] D. Chae, O. Imanuvilov, The existence of non-topological multivortex solutions in the relativistic self-dual Chern-Simons theory, Comm. Math. Phys. 215 (2000) 119-142.

[12] D. Chae, G. Tarantello, On planar electroweak vortices, Ann. Inst. H. Poincaré Analyse Non Linéaire, in press.

[13] S. Chandrasekhar, An Introduction to the Study of Stellar Structure, Dover, New York, 1957.

[14] W. Chen, C. Li, Classification of solutions of some nonlinear elliptic equations, Duke Math. J. 63 (1991) 615-623.

[15] C.C. Chen, C.S. Lin, Topological degree for a mean field equation on Riemann surfaces, Comm. Pure Appl. Math. 56 (2003) $1667-1727$.

[16] C.C. Chen, C.S. Lin, On the simmetry of blowup solutions to a mean field equation, Ann. Inst. H. Poincaré Analyse Non Linéaire 18 (2001) 271-296.

[17] M. Del Pino, M. Kowalczyk, M. Musso, Singular limits in Liouville-type equation, preprint.

[18] E.N. Dancer, On the uniqueness of the positive solution of a singularly perturbed problem, Rocky Mountain J. Math. 25 (1995) 957-975.

[19] W. Ding, J. Jost, J. Li, G. Wang, Existence results for mean field equations, Ann. Inst. H. Poincaré Analyse Non Linéaire 16 (1999) 653-666.

[20] K. El Mehdi, M. Grossi, Asymptotic estimates and qualitative properties of an elliptic problem in dimension two, preprint.

[21] P. Esposito, Blow up solutions for a Liouville equation with singular data, preprint, 2003.

[22] P. Esposito, A class of Liouville-type equations arising in Chern-Simons vortex theory: asymptotics and construction of blowing up solutions, Thesis, Roma "Tor Vergata", 2003.

[23] I.M. Gelfand, Some problems in the theory of quasilinear equations, Amer. Math. Soc. Transl. 29 (1969) 295-381.

[24] D. Gilbarg, N.S. Trudinger, Elliptic Partial Differential Equations of Second Order, second ed., Springer-Verlag, 1983.

[25] M. Grossi, A. Pistoia, On the effect of critical points of distance function in superlinear elliptic problems, Adv. Differential Equations 5 (2000) 1397-1420.

[26] Y.Y. Li, On a singularly perturbed elliptic equation, Adv. Differential Equations 2 (1997) 955-980.

[27] J. Liouville, Sur l'équation aud dérivées partielles $\partial^{2} \log \lambda / \partial u \partial v \pm 2 \lambda a^{2}=0$, J. Math. 18 (1853) 71-72.

[28] L. Ma, J. Wei, Convergence for a Liouville equation, Comment. Math. Helv. 76 (2001) 506-514.

[29] N. Mizoguchi, T. Suzuki, Equations of gas combustion: $S$-shaped bifurcation and mushrooms, J. Differential Equations 134 (1997) $183-$ 215.

[30] J.L. Moseley, Asymptotic solutions for a Dirichlet problem with an exponential nonlinearity, SIAM J. Math. Anal. 14 (1983) 719-735.

[31] J.L. Moseley, A two-dimensional Dirichlet problem with an exponential nonlinearity, SIAM J. Math. Anal. 14 (1983) $934-946$. 
[32] J. Moser, A sharp form of an inequality by N. Trudinger, Indiana Univ. Math. J. 20 (1970/71) 1077-1092.

[33] M. Musso, A. Pistoia, Multispike solutions for a nonlinear elliptic problem involving the critical Sobolev exponent, Indiana Univ. Math. J. 51 (2002) 541-579.

[34] J.D. Murrey, Mathematical Biology, Springer, Berlin, 1989.

[35] K. Nagasaki, T. Suzuki, Asymptotic analysis for a two dimensional elliptic eigenvalue problem with exponentially dominated nonlinearity, Asymptotic Anal. 3 (1990) 173-188.

[36] M. Nolasco, Non-topological $N$-vortex condensates for the self-dual Chern-Simons theory, Comm. Pure Appl. Math. 56 (2003) $1752-$ 1780.

[37] O. Rey, The role of Green's function in a nonlinear elliptic equation involving the critical Sobolev exponent, J. Funct. Anal. 89 (1990) $1-52$.

[38] T. Suzuki, Two dimensional Emden-Fowler equation with exponential nonlinearity, Nonlinear Diffusion Equations and their Equilibrium States 3 (1992) 493-512.

[39] T. Suzuki, Global analysis for a two-dimensional eigenvalue problem with exponential nonlinearity, Ann. Inst. H. Poincaré Analyse Non Linéaire 9 (1992) 367-398.

[40] N.S. Trudinger, On imbeddings into Orlicz spaces and some applications, J. Math. Mech. 17 (1967) $473-483$.

[41] V.H. Weston, On the asymptotic solution of a partial differential equation with exponential nonlinearity, SIAM J. Math. Anal. 9 (1978) 1030-1053. 